\title{
Direct simulation vs volume-averaged treatment of adiabatic, premixed flame in a porous medium
}

\author{
M. SAHRAOUI and M. KAVIANY $\uparrow$ \\ Department of Mechanical Engineering and Applied Mechanics, University of Michigan, \\ Ann Arbor, MI 48109, U.S.A.
}

(Received 1 October 1993 and in final form 3 May 1994)

\begin{abstract}
For the case of flame thickness being of the order of the pore linear dimension, the flame structure and speed in adiabatic, premixed methane-air combustion in porous media are examined. The local, volume-averaged conservation equations that assume a local thermal equilibrium between the solid and the gas phases (i.e. the single-medium treatment) or allow for a thermal nonequilibrium (i.e. the twomedium treatment) are used along with the direct application of the pointwise conservation equations to a two-dimensional porous medium model (ordered arrangement of discrete or connected square cylinders). The effective properties of the porous medium in the volume-averaged treatments, including the interfacial Nusselt number, are found by applying the local volume-averaging principles. The results show that, although significant variations of the temperature and species concentrations occur over a pore, the flame structure, thickness, speed, and excess temperature (i.e. local gas temperature in excess of the adiabatic temperature) are fairly well predicted by the two-medium model (the single-medium treatment is unable to predict the local excess temperature). However, the volume-averaged treatments are unable to predict the pore-level, local high temperature region in the gas phase (which can be up to $40 \%$ above the adiabatic temperature), and the pore-level variation in the flame speed with respect to the flame location in the pore (which can vary by up to $20 \%$ ). Other shortcomings of the volume-averaged treatments are also revealed through a parametric examination involving the pore-geometry variables, solid to gas conductivity ratio, equivalence ratio, porosity, and flame location within the pore.
\end{abstract}

\section{INTRODUCTION}

Recently there has been a growing intercst in porous radiant burners (nonadiabatic) for efficient radiative heat transfer and for extending the range of the flame speed and the flammability of lean mixtures of hydrocarbons (mostly methane-air). Nonadiabatic porous burners result in low $\mathrm{NO}_{x}$ and $\mathrm{CO}$ emissions. These advantageous features of porous burners are primarily due to the enhanced conductive and radiative properties of the solid matrix which allow for the enhanced preheating of the mixture. This enhanced preheating results in a higher burning speed (compared to the adiabatic flame speed in the gas alone, i.e. plain media). This enhanced preheating also allows for burning of lean mixtures which are otherwise nonflammable. Applications involving combustion in porous media were discussed by Echigo [1] and Hanamura et al. [2].

Takeno and Sato [3] performed an analytical and numerical study of the adiabatic combustion in porous media using a simple two-medium thermal model which allows for a thermal nonequilibrium between the gas and the solid phases, but assumes that the temperature of the porous solid is constant and equal to the adiabatic flame temperature. They found

†Author to whom correspondence should be addressed. that, in the porous medium, the adiabatic flame speed is higher than that in plain media, $u_{\mathrm{F}_{\mathrm{n}}}$. They also found that the flame structure and speed are sensitive to the magnitude of the interfacial Nusselt number between the solid and the gas. This was extended by Takeno et al. [4] to include a finite-length porous medium and they found that the solid temperature can have two different values for the same flow rate. This behavior is due to the unrealistic assumption that the solid temperature is constant. For a flame speed that is very close to that of the adiabatic flame in plain media, the reaction zone is very close to the upstream boundary of the porous medium.

Further improvements to the models mentioned above were made by Takeno and Hase [5] who considered nonadiabatic premixed combustion. They found that as the heat loss is increased, the upper limit for the flame speed is decreased and the lower limit is increased. However, this model is approximate and provides a qualitative idea about the energy feedback due to conduction through a porous medium with high conductivity. Yoshizawa et al. [6] considered the nonadiabatic, premixed flame in a finite-length porous medium by using a two-medium thermal treatment, and included the effect of radiation. Their numerical results show that the flame speed may be an order of magnitude larger than the adiabatic flame speed $u_{F_{0}}$, depending on the radiative and conductive effective 


\section{NOMENCLATURE}

$A \quad$ area $\left[\mathrm{m}^{2}\right]$ or pre-exponential factor $\left[\mathrm{s}^{-1}\right]$

$c \quad$ thickness of connecting arm [m]

$c_{\mathrm{p}}$ specific heat capacity $\left[\mathrm{J} \mathrm{kg}^{-1} \mathrm{~K}^{-1}\right.$ ]

$d$ linear dimension of solid phase in unit cell $[\mathrm{m}]$

$D \quad$ mass diffusion coefficient $\left[\mathrm{m}^{2} \mathrm{~s}^{-1}\right]$

$D^{\mathrm{d}} \quad$ hydrodynamic dispersion coefficient $\left[\mathrm{m}^{2} \mathrm{~s}^{-1}\right]$

$h_{\mathrm{sg}}$ heat transfer coefficient $\left[\mathrm{W} \mathrm{m} \mathrm{m}^{-2} \mathrm{~K}^{-1}\right]$

$k$ conductivity [ $\mathrm{W} \mathrm{m}^{-1} \mathrm{~K}^{-1}$ ]

$l \quad$ unit-cell dimension [m]

$L e_{\mathrm{m}}$ molecular Lewis number

$L e_{\mathrm{e}_{1}} \quad$ single-medium effective Lewis number

$L e_{\mathrm{e}_{2}} \quad$ two-medium effective Lewis number

n normal unit vector

$N u_{1} \quad$ Nusselt number, $h_{\mathrm{sg}} l / k_{\mathrm{g}}$

$\dot{n} \quad$ volumetric production rate of product species $\left[\mathrm{kg} \mathrm{m}^{-3} \mathrm{~s}^{-1}\right]$

$P e_{\mathrm{I}} \quad$ Péclet number, $u_{\mathrm{F}} l / \alpha_{\mathrm{g}}$

$p \quad$ pressure $\left[\mathrm{N} \mathrm{m}^{-2}\right]$

$\operatorname{Pr} \quad$ Prandtl number, $v / \alpha_{\mathrm{g}}$

$R_{\mathrm{g}} \quad$ universal gas constant, $8.314 \mathrm{~J} \mathrm{~mol}^{-1}$ $\mathrm{K}^{-1}$

$R e_{1} \quad$ Reynolds number, $u_{\mathrm{F}} l / v$

$T \quad$ local temperature [K]

$\langle T\rangle_{y}$ local line-averaged temperature in $y$-direction, $\langle T\rangle_{y}(x, y)=$ $(1 / l) \int_{-l / 2}^{l / 2} T\left(x, y+y^{\prime}\right) \mathrm{d} y^{\prime}[\mathrm{K}]$

$\langle T\rangle$ local volume-averaged temperature, $V^{-1} \int_{V} T \mathrm{~d} V[\mathrm{~K}]$

$\langle T\rangle^{i} \quad$ local phase $i(i=\mathrm{g}$ or $\mathrm{s})$ volume-averaged temperature $V_{i}^{-1} \int_{V_{i}} T_{i} \mathrm{~d} V[\mathrm{~K}]$

$\mathbf{u} \quad$ velocity vector $\left[\mathrm{m} \mathrm{s}^{-1}\right]$

$u, v \quad$ velocity in $x$-and $y$-direction $\left[\mathrm{m} \mathrm{s}^{-1}\right]$

$V \quad$ local representative elementary volume $\left[\mathrm{m}^{3}\right]$

$x, y \quad$ Cartesian coordinate axes [m]
$x_{\mathrm{F}} \quad$ flame location [mm]

$Y$ mass fraction of product species.

Greek symbols

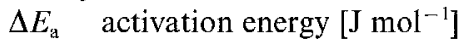

$\Delta i_{\mathrm{c}} \quad$ specific heat of combustion [ $\mathrm{J} \mathrm{kg}^{-1}$ ]

$\Delta T_{\max }^{*}$ dimensionless excess temperature, $\left(T_{\max }-T_{\mathrm{a}}\right) /\left(T_{\mathrm{a}}-T_{\mathrm{n}}\right)$

$\varepsilon \quad$ porosity, $1-d^{2} / l^{2}$

$\mu \quad$ dynamic viscosity $\left[\mathrm{kg} \mathrm{m}^{-1} \mathrm{~s}^{-1}\right]$

$v \quad$ kinematic viscosity $\left[\mathrm{m}^{2} \mathrm{~s}^{-1}\right]$

$\rho \quad$ density $\left\lfloor\mathrm{kg} \mathrm{m}^{-3}\right\rfloor$

$\Phi$ equivalence ratio.

\section{Subscripts}

A area

a adiabatic

D Darcean

$F \quad$ flame or fuel

$\mathrm{F}_{\mathrm{o}} \quad$ flame in plain media

g gas

m mass or molecular

$\max$ maximum

n nonreacted

o plain medium or oxidant

$\mathrm{p}$ products

s solid or surface

$\mathrm{V}$ volume

$x \quad x$-direction

$y \quad y$-direction.

Superscripts

b bulk

F flame

g gas

s solid.

Other

$\langle$ volume- or line-averaged. properties of the porous medium. Sathe et al. [7, 8] also showed that the flame speed depends significantly on the flame location and the optical thickness of the porous slab, and, for the porous medium and equivalence ratio $\Phi$ used, they showed an up to sevenfold increase in speed over $u_{\mathrm{F}_{0}}$. A discussion of the nonadiabatic premixed combustion in porous media was given by Kaviany [9].

The existing studies of premixed gaseous combustion in porous media use one-dimensional, approximate local phase-volume-averaged energy and species conservation equations, and the necessary constitutive equations for the effective properties. The change in the pressure across the flame is generally negligible compared to the absolute pressure, and the pressure is assumed constant. For porous media with random structure, the local phase-volume-averaged energy and species equations are derived using representative, phasic elementary volumes with a linear dimension $l$ which is much larger than the small length scale (i.e. the diameter of particles $d$ ), but much smaller than the system linear dimension $L$, i.e.

$$
d \ll l \ll L .
$$

These local volume-averaged equations are derived assuming that the temperature and concentration variations across the small length scale $d$ is much smaller than that across $L$, i.e. $\Delta T_{\mathrm{d}} \ll \Delta T_{\mathrm{L}}$ [10]. However, in hydrocarbon-air combustion in porous media, the flame thickness can be of the order of magnitude 
of $d$, and, therefore, the accuracy of the averaged equations in predicting the flame structure has been questioned. In addition, the effective properties, such as the effective molecular and radiant conductivity, mass diffusivity, and hydrodynamic dispersion coefficient, must be prescribed with relatively high accuracy.

In this study, we perform a direct numerical simulation of the premixed combustion in a two-dimensional model poroủs medium made of in-line or staggered arrangements of connected or discrete square cylinders. This two-dimensional numerical simulation allows for the study of the effect of the pore-level hydrodynamics and heat and mass transport on the flame structure and speed. A very low solid surface emmisivity is assumed, and, therefore, the effect of the radiative heat transfer is assumed to be negligible. The results of this direct simulation are also compared with the results of the volume-averaged treatments (single- and two-medium), and the accuracy of the volume-averaged models, in predicting the flame structure and speed, is examined.

\section{TWO-DIMENSIONAL DIRECT SIMULATION}

The two-dimensional porous medium model, the pointwise (i.e. pore-level) direct simulation, and the local volume-averaged models (single- and two-medium treatments) are described below. In the volumeaveraged treatments, the effective conductivity and the axial hydrodynamic dispersion coefficient are those obtained previously by Sahraoui and Kaviany [11, 12].

\subsection{Pointwise description}

Direct simulations of the premixed methane-air combustion are made for the in-line or staggered arrangements of connected or discrete square cylinders as shown in Fig. 1. The continuity equation for the gas phase is given by

$$
\frac{\partial \rho_{\mathrm{g}}}{\partial t}+\frac{\partial \rho_{\mathrm{g}} u}{\partial x}+\frac{\partial \rho_{\mathrm{g}} v}{\partial y}=0 .
$$

The density of the gas phase $\rho_{\mathrm{g}}$ is given by the ideal gas law at atmospheric pressure (inlet pressure) and variable local temperature. In the momentum equation, we assume that the gas viscosity is constant and that the buoyancy effect is negligible, and write

$$
\begin{gathered}
\rho_{\mathrm{g}} \frac{\partial u}{\partial t}+\rho_{\mathrm{g}} u \frac{\partial u}{\partial x}+\rho_{\mathrm{g}} v \frac{\partial u}{\partial y}=-\frac{\partial p}{\partial x}+\mu\left(\frac{\partial^{2} u}{\partial x^{2}}+\frac{\partial^{2} u}{\partial y^{2}}\right) \\
\rho_{\mathrm{g}} \frac{\partial v}{\partial t}+\rho_{\mathrm{g}} u \frac{\partial v}{\partial x}+\rho_{\mathrm{g}} v \frac{\partial v}{\partial y}=-\frac{\partial p}{\partial y}+\mu\left(\frac{\partial^{2} v}{\partial x^{2}}+\frac{\partial^{2} v}{\partial y^{2}}\right)
\end{gathered}
$$

In the energy equations, the specific heat capacity $c_{\mathrm{p}}$ and conductivity $k_{\mathrm{g}}$ are assumed constant and evaluated at the average temperature (i.e. $1300 \mathrm{~K}$ for an equivalence ratio of unity). The gas-phase energy equation is written as

$$
\begin{gathered}
\left(\rho c_{\mathrm{p}}\right)_{\mathrm{g}} \frac{\partial T_{\mathrm{g}}}{\partial t}+\left(\rho c_{\mathrm{p}}\right)_{\mathrm{g}} u \frac{\partial T_{\mathrm{g}}}{\partial x}+\left(\rho c_{\mathrm{p}}\right)_{\mathrm{g}} v \frac{\partial T_{\mathrm{g}}}{\partial y}=\frac{\partial}{\partial x} k_{\mathrm{g}} \frac{\partial T_{\mathrm{g}}}{\partial x} \\
+\frac{\partial}{\partial y} k_{\mathrm{g}} \frac{\partial T_{\mathrm{g}}}{\partial y}+\dot{n} \Delta i_{\mathrm{c}} \quad \text { in } V_{\mathrm{g}}
\end{gathered}
$$

where $\dot{n}$ is the volumetric rate of generation of product species and $\Delta i_{c}$ is the specific heat of combustion. The solid-phase energy equation is

$$
\left(\rho c_{\mathrm{p}}\right)_{\mathrm{s}} \frac{\partial T_{\mathrm{s}}}{\partial t}=\frac{\partial}{\partial x} k_{\mathrm{s}} \frac{\partial T_{\mathrm{s}}}{\partial x}+\frac{\partial}{\partial y} k_{\mathrm{s}} \frac{\partial T_{\mathrm{s}}}{\partial y} \quad \text { in } V_{\mathrm{s}} .
$$

The species conservation equation is written for the product species as

$$
\begin{aligned}
\rho_{\mathrm{g}} \frac{\partial Y}{\partial t}+\rho_{\mathrm{g}} u \frac{\partial Y}{\partial x}+\rho_{\mathrm{g}} v \frac{\partial Y}{\partial y}=\frac{\partial}{\partial x} & D \rho_{\mathrm{g}} \frac{\partial Y}{\partial x} \\
& +\frac{\partial}{\partial y} D \rho_{\mathrm{g}} \frac{\partial Y}{\partial y}+\dot{n}
\end{aligned}
$$

where $Y=\rho_{\mathrm{p}, \mathrm{g}} / \rho_{\mathrm{g}}$ is the mass fraction of the product species. For the species diffusivity, we assume that the molecular Lewis number, $L e_{\mathrm{m}}=D / \alpha_{\mathrm{g}}$, is unity. For the species generation term, a first-order, Arrhenius relation given by Yoshizawa et al. [6] is used, i.e.

$$
\dot{n}=A \rho_{\mathrm{g}}(1-Y) \exp \left(-\Delta E_{\mathrm{a}} / R_{\mathrm{g}} T_{\mathrm{g}}\right) .
$$

The boundary conditions at the inlet of the computational domain are

$$
\begin{gathered}
u=u_{\mathrm{F}} \quad v=0 \quad T_{\mathrm{g}}=T_{\mathrm{n}} \\
\text { and } Y=0 \text { for } x \rightarrow-\infty
\end{gathered}
$$

and, for the exit,

$$
\begin{gathered}
\rho_{\mathrm{g}} u=\rho_{\mathrm{g}}\left(T_{\mathrm{n}}\right) u_{\mathrm{r}} \quad v=0 \quad \frac{\partial T_{\mathrm{g}}}{\partial x}=0 \\
\text { and } \frac{\partial Y}{\partial x}=0 \quad \text { for } \quad x \rightarrow \infty
\end{gathered}
$$

As mentioned above, the inlet pressure is assumed to be atmospheric. By trial and error, the exit and inlet boundaries are located far enough from the flame, where the effect of any further increase in the computational domain upstream and downstream does not change the results. At the upper and lower boundaries of the domain, symmetry conditions are used for all the quantitics. At the solid-gas interface, the noslip condition for velocity, i.e.

$$
u=v=0 \quad \text { on } \quad A_{\mathrm{gs}}
$$

is used. For the temperature, assuming that the surface emissivity of the solid is very low [for opaque nonabsorbing particles, the radiant conductivity will have a threshold value which is due to scattering (Kaviany and Singh [13]); here we assume that this threshold value is negligible and in the direct simulation and the volume-averaged treatments the radiation con- 
(a) Unit Cell

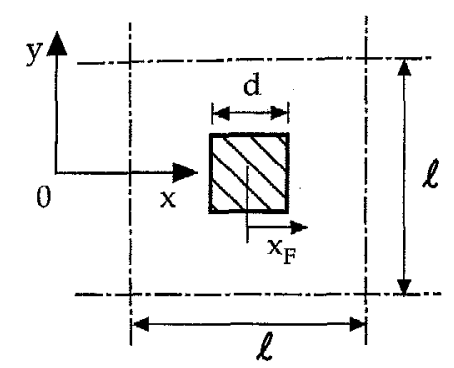

(b) Staggered Arrangement

(c) Interparticle Connection

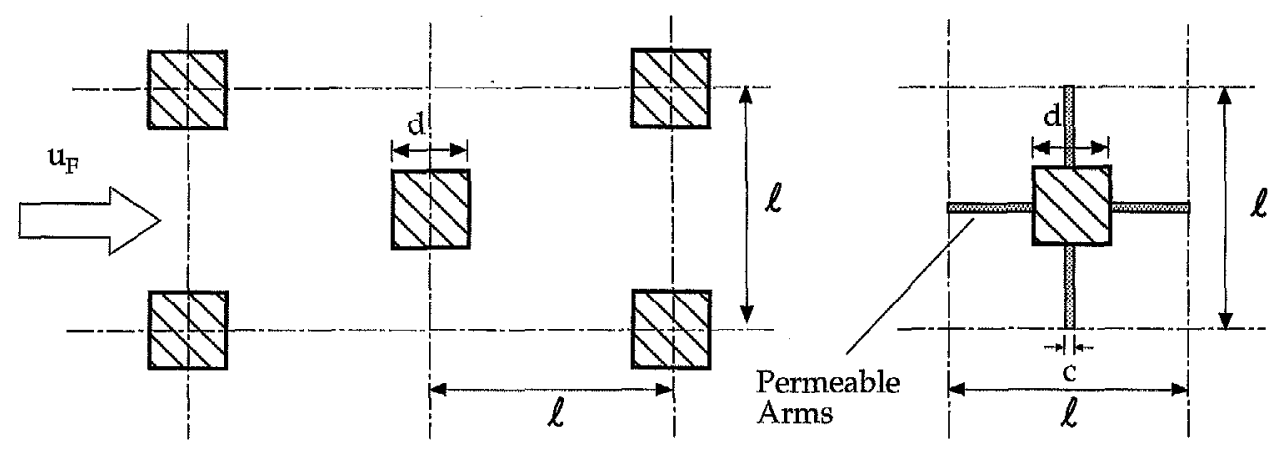

Fig. 1. Various arrangements of square cylinders used in the two-dimensional simulations: (a) discrete solid phase with in-line arrangement, (b) discrete solid phase with staggered arrangement, and (c) continuous solid phase with in-line arrangement.

tribution is neglected], the temperature boundary conditions become

$$
T_{\mathrm{s}}=T_{\mathrm{g}} \quad k_{\mathrm{s}} \mathbf{n} \cdot \nabla T_{\mathrm{s}}=k_{\mathrm{g}} \mathbf{n} \cdot \nabla T_{\mathrm{g}} \quad \text { on } \quad A_{\mathrm{gs}}
$$

Here the radiative heat transfer is not addressed because its inclusion would require a substantial increase in the computational domain (i.e. number of unit cells used in the simulation). This increases both the CPU and the memory requirements. In addition, the surface emissivity and the extent of the surfaceproperty direction dependence has to be addressed, and would have added substantially to the complexity. For the species, the condition of no mass flux is used, i.e.

$$
\mathrm{n} \cdot \nabla Y=0 \text { on } A_{\mathrm{gs}} .
$$

The above equations are solved for a variable equivalence ratio $\Phi$ which is defined as

$$
\Phi=\frac{\left(\frac{v_{\mathrm{F}}}{v_{0}}\right)_{\text {actual }}}{\left(\frac{v_{\mathrm{F}}}{v_{0}}\right)_{\text {stoich. }}}
$$

where $v_{\mathrm{F}}$ and $v_{0}$ are the molar coefficients for the fuel and the oxidant in the stoichiometric reaction equation.

\subsection{Continuous and discrete solid phase}

In most applications of porous media, the solid phase is continuous and heat conduction through the solid is a very important mechanism of heat transfer. Therefore, modeling this aspect of the porous medium is very important in order to obtain an accurate prediction of the flame structure and speed. In the twodimensional simulation, the continuous solid phase is modeled by connecting the neighboring particles by connecting arms, as depicted in Fig. 1(c). This approach is used by Nozad et al. [14] in order to model the effective conductivity of randomly packed beds of spherical particles. The connecting arms will have the same conductivity as the solid, but are permeable (with large permeability).

\subsection{Nusselt number}

In the two-medium treatment, the heat exchange between the two media is modeled using a prescribed Nusselt number relation and the temperature difference between the two phases. This Nusselt number 
for the in-line and staggered arrangements of square cylinders is computed here. The continuity and momentum equations, equations (2)-(4), are solved along with the energy equation, equation (5), along with $\dot{n}=0$ and a prescribed solid surface temperature. In the energy equation, we solve for the dimensionless temperature $T^{*}$ defined as

$$
T^{*}=\frac{T_{\mathrm{g}}-T_{\mathrm{n}}}{T_{\mathrm{s}}-T_{\mathrm{n}}}
$$

In these computations, the gas is assumed to be incompressible. The boundary conditions at the inlet and exit, for velocity and temperature, are those given in equations (9) and (10), with $T_{\mathrm{n}}^{*}=0$. The surface temperature of all cylinders is constant, i.e. $T_{s}^{*}=1$. The surface-area-average Nusselt number is found by area averaging the local Nusselt number on a cylinder, i.e.

$$
\left\langle N u_{1}\right\rangle_{A_{\mathrm{gs}}}=-\frac{1}{A_{\mathrm{gs}}} \int^{A_{\mathrm{gs}}} \frac{l}{T_{\mathrm{s}}-\langle T\rangle_{y}^{\mathrm{b}}} \nabla T_{\mathrm{g}} \cdot \mathbf{n} \mathrm{d} A
$$

where $\langle T\rangle_{y}^{\mathrm{b}}$ is the local, bulk mixed gas temperature defined as

$$
\langle T\rangle_{y}^{\mathrm{b}}=\frac{\int_{-l / 2}^{\ell / 2} \rho_{\mathrm{g}} u(x, y) T_{\mathrm{g}}(x, y) \mathrm{d} y}{\int_{-l / 2}^{l / 2} \rho_{\mathrm{g}} u(x, y) \mathrm{d} y} .
$$

The computed results showing the variation of the Nusselt number with respect to the particle Reynolds number, particle arrangement, and porosity are discussed in Section 4.2.

\subsection{Solution method}

The momentum, energy, and species equations are written in the finite-volume form as discussed by Patankar [15]. The staggered grid is used for the velocity and pressure, and the implicit method is used for the time dependence. Convergence in each time step is obtained by evaluating the residues in the continuity, energy, and species equations, and checking that their magnitude is below a preseribed valuc. Only stcadystate solutions are sought and steps are taken to facilitate the approach to the steady state. After every time step, the flame velocity is adjusted using the conservation of species by integrating equation (7) over the entire domain, i.e.

$$
\rho_{\mathrm{g}} u_{\mathrm{F}}=\frac{2}{l} \int_{0}^{l / 2} \int_{-\infty}^{\infty} \dot{n} \mathrm{~d} x \mathrm{~d} y .
$$

The overall convergence is obtained when a six-digit accuracy in the flame speed $u_{\mathrm{F}}$ is obtained. Progressively smaller grids are used until the flame speed is changed by $<1 \%$ (the one-dimensional volumeaveraged equations are used for selection of the initial grid size in the $x$-direction). For most solutions, the initial guess is provided from the previous results, and, whenever such results are not available, the temperature and species distributions are obtained from the solution to the one-dimensional volume-averaged equations. For the two-dimensional results, the flame location $x_{\mathrm{F}}$ is assigned to the grid point having the maximum reaction rate, and this occurs at $y / l=0.5$ for the in-line arrangement. During the iteration, whenever the flame moves from the prescribed axial location, in the next time step the entire velocity, pressure, temperature, and species fields are moved back.

In order to find the numerical values of the preexponential factor $A$ and the activation energy $\Delta E_{\mathrm{a}}$ in equation (8), the approach of Yoshizawa et al. [6] is used. The flame velocity is matched with the experimental data of the one-dimensional adiabatic flame speed at two equivalence ratios (i.e. $\Phi=1$ and 0.5 ). For methane-air, the flame speed for $\Phi=1$ is 38.2 $\mathrm{cm} \mathrm{s}^{-1}$ and for $\Phi=0.5$ it is $4.15 \mathrm{~cm} \mathrm{~s}^{-1}$. Using this approach we find that $A$ is $2.2 \times 10^{8} \mathrm{~s}^{-1}$ and $\Delta E_{\text {a }}$ is $141 \times 10^{3} \mathrm{~J} \mathrm{~mol}^{-1}$. The flame velocity prediction for the one-dimensional premixed flame is also compared with the numerical results of Mauss and Peters [16], who used a multiple-step kinetics. For equivalence ratios of 0.5 and 1 , a good agreement is found for the flame speed. The flame speed for $\Phi=0.7\left(u_{\mathrm{F}}=14.1\right.$ $\mathrm{cm} \mathrm{s}^{-1}$ ) is also compared with the results of Mauss and Peters [16] and a good agreement is obtained.

\section{VOLUME-AVERAGED MODELS}

The local volume-averaged models used in the studies of combustion in porous media are the two-medium treatment which allows for a thermal nonequilibrium between the phases, and the singlemedium treatment which assumes a local thermal equilibrium.

\subsection{Two-medium treatment}

The two-medium thermal and species models used here are [9]

$$
\begin{aligned}
& \varepsilon\left(\rho c_{\mathrm{p}}\right)_{\mathrm{g}} \frac{\partial\langle T\rangle^{\mathrm{g}}}{\partial t} \\
& +\left(\rho c_{\mathrm{p}}\right)_{\mathrm{g}} u_{\mathrm{F}} \frac{\partial\langle T\rangle^{\mathrm{g}}}{\partial x}=\frac{\partial}{\partial x}\left\{\varepsilon\left[\langle k\rangle^{\mathrm{g}}+\left(\rho c_{\mathrm{p}}\right)_{\mathrm{g}} D_{x x}^{\mathrm{d}}\right] \frac{\partial\langle T\rangle^{\mathrm{g}}}{\partial x}\right\} \\
& +\frac{A_{\mathrm{gs}}}{V}\left\langle N u_{\mathrm{l}}\right\rangle_{A_{\mathrm{gs}}} \frac{k_{\mathrm{g}}}{l}\left(\langle T\rangle^{\mathrm{s}}-\langle T\rangle^{\mathrm{g}}\right) \\
& +\varepsilon\langle\dot{n}\rangle^{\mathrm{g}} \Delta i_{\mathrm{c}} \\
& \left.(1-\varepsilon)\left(\rho c_{\mathrm{p}}\right)_{\mathrm{s}} \frac{\partial\langle T\rangle^{\mathrm{s}}}{\partial t}=\frac{\partial}{\partial x} \mid(1-\varepsilon)\langle k\rangle^{\mathrm{s}} \frac{\partial\langle T\rangle^{\mathrm{s}}}{\partial x}\right] \\
& \quad+\frac{A_{\mathrm{g}}}{V}\left\langle N u_{1}\right\rangle_{A_{\mathrm{gs}}} \frac{k_{\mathrm{g}}}{l}\left(\langle T\rangle^{\mathrm{g}}-\langle T\rangle^{\mathrm{s}}\right) \\
& \varepsilon \rho_{\mathrm{g}} \frac{\partial\langle Y\rangle^{\mathrm{g}}}{\partial t}+\rho_{\mathrm{g}} u_{\mathrm{F}} \frac{\partial\langle Y\rangle^{\mathrm{g}}}{\partial x} \\
& \quad \frac{\partial}{\partial x}\left[\varepsilon\left(\langle D\rangle^{\mathrm{g}}+D_{\mathrm{m}_{x x}}^{\mathrm{d}}\right) \rho_{\mathrm{g}} \frac{\partial\langle Y\rangle^{\mathrm{g}}}{\partial x}\right]+\varepsilon\langle\dot{n}\rangle^{\mathrm{g}} .
\end{aligned}
$$


Table 1. Correlations for the hydrodynamic dispersion $D_{x x}^{\mathrm{d}} / \alpha_{\mathrm{g}}$ as a function of $P e_{1}$ for $\varepsilon=0.8$ and 0.9

\begin{tabular}{ccc}
\hline & \multicolumn{2}{c}{$D_{x x}^{\mathrm{d}} / \alpha_{\mathrm{g}}$} \\
\cline { 2 - 3 }$P e_{1}$ & $\varepsilon=0.8$ & $\varepsilon=0.9$ \\
\hline$P e_{1}<1$ & 0 & 0 \\
$1<P e_{1}<10$ & $0.128\left(P e_{1}-1\right)$ & $0.071\left(P e_{1}-1\right)$ \\
$10 \leqslant P e_{1} \leqslant 10^{3}$ & $0.019 P e_{1}^{1.82}$ & $0.009 P e_{1}^{1.86}$ \\
\hline
\end{tabular}

For the momentum equation, the fluid flow through porous media is governed by Darcy's law. Assuming that the pressure drop in the porous medium is negligible, only the volume-averaged continuity equation is needed, which implies that $\rho_{\mathrm{g}} u_{\mathrm{F}}$ is constant for this volume-averaged, one-dimensional flow.

The effective conductivities of the solid and gas phases are determined using the equivalent thermalcircuit model. For the discrete solid phase, the gas phase is assumed to conduct heat through a continuous phase existing between any two adjacent square cylinders, i.e.

$$
\varepsilon\langle k\rangle^{\mathrm{g}}=k_{\mathrm{g}}\left[1-(1-\varepsilon)^{1 / 2}\right] .
$$

For the solid, the heat is assumed to be conducted through the remaining volume, i.e.

$$
(1-\varepsilon)\langle k\rangle^{\mathrm{s}}=\frac{(1-\varepsilon)^{1 / 2} k_{\mathrm{s}} k_{\mathrm{g}}}{\left[1-(1-\varepsilon)^{1 / 2}\right] k_{\mathrm{s}}+(1-\varepsilon)^{1 / 2} k_{\mathrm{g}}} .
$$

For the continuous solid phase, we have

$$
\begin{gathered}
(1-\varepsilon)\langle k\rangle^{s}=\frac{\left[(1-\varepsilon)^{1 / 2}-\frac{c}{a}(1-\varepsilon)^{1 / 2}\right] k_{\mathrm{s}} k_{\mathrm{g}}}{\left[1-(1-\varepsilon)^{1 / 2}\right] k_{\mathrm{s}}+(1-\varepsilon)^{1 / 2} k_{\mathrm{g}}} \\
+\frac{c}{a}(1-\varepsilon)^{1 / 2} k_{\mathrm{s}}
\end{gathered}
$$

and, for the gas phase, the effective conductivity is given by

$$
\varepsilon\langle k\rangle^{\mathrm{g}}=\frac{\left[1-(1-\varepsilon)^{1 / 2}\right] k_{\mathrm{s}} k_{\mathrm{g}}}{\left[1-\frac{c}{a}(1-\varepsilon)^{1 / 2}\right] k_{\mathrm{s}}+\frac{c}{a}(1-\varepsilon)^{1 / 2} k_{\mathrm{g}}} .
$$

For the axial hydrodynamic thermal dispersion coefficient $D_{x x}^{\mathrm{d}}$, we use the results from Sahraoui and Kaviany [12] for the in-line arrangement of circular cylinders. This is a good approximation, since the particle shape does not affect the hydrodynamic dispersion significantly (especially for low Péclet numbers). The correlations for $D_{x x}^{\mathrm{d}} / \alpha_{\mathrm{g}}$ as a function of the Péclet number are given in Table 1. For the effective properties in the species equation, we assume that the effective mass diffusivity is the same as the thermal diffusivity of the gas. Also, we assume that the hydrodynamic dispersion for the heat and mass diffusion are the same. Note that $D_{x x}^{\mathrm{d}}$ depends on $k_{\mathrm{s}} / k_{\mathrm{g}}$ (e.g. ref. [12]) and that the axial, species hydrodynamic dispersion coefficient $D_{\mathrm{m}_{x x}}^{\mathrm{d}}$ is the same as $D_{x x}^{\mathrm{d}}$, only if $k_{\mathrm{s}} / k_{\mathrm{g}}=0$. Here, the effective, two-medium Lewis number, assumed to be unity, becomes

$$
L e_{\mathrm{e}_{2}}=\frac{\frac{\langle k\rangle^{\mathrm{g}}}{\left(\rho c_{\mathrm{p}}\right)_{\mathrm{g}}}+D_{x x}^{\mathrm{d}}}{\langle D\rangle^{\mathrm{g}}+D_{\mathrm{m}_{x x}}^{\mathrm{d}}}=1
$$

For the boundary conditions, at the inlet and exit a local thermal equilibrium is assumed. The boundary conditions for the phase-averaged temperature and mass fraction at the inlet are

$$
\langle T\rangle^{\mathrm{g}}=\langle T\rangle^{\mathrm{s}}=T_{\mathrm{n}} \quad\langle Y\rangle^{\mathrm{g}}=0 \text { for } x \rightarrow-\infty
$$

and, at the exit,

$$
\frac{\partial\langle T\rangle^{\mathrm{g}}}{\partial x}=\frac{\partial\langle T\rangle^{\mathrm{s}}}{\partial x}=\frac{\partial\langle Y\rangle^{\mathrm{g}}}{\partial x}=0 \text { for } x \rightarrow \infty .
$$

\subsection{Single-medium treatment}

For the single-medium thermal model, i.e. where a local thermal equilibrium (i.e. $\langle T\rangle^{\mathrm{s}}=\langle T\rangle^{\mathrm{g}}=\langle T\rangle$ ) between the phases is assumed, equations (19) and (20) are added to obtain

$$
\begin{aligned}
& {\left[\varepsilon\left(\rho c_{\mathrm{p}}\right)_{\mathrm{g}}+(1-\varepsilon)\left(\rho c_{\mathrm{p}}\right)_{\mathrm{s}}\right] \frac{\partial\langle T\rangle}{\partial t}} \\
& +\left(\rho c_{\mathrm{p}}\right)_{\mathrm{g}} u_{\mathrm{F}} \frac{\partial\langle T\rangle}{\partial x}=\frac{\partial}{\partial x}\left\{\left[\left\langle k_{\mathrm{e}}\right\rangle+\varepsilon\left(\rho c_{\mathrm{p}}\right)_{\mathrm{g}} D_{x_{x}}^{\mathrm{d}}\right] \frac{\partial\langle T\rangle}{\partial x}\right\} \\
& +\varepsilon\langle\dot{n}\rangle^{\mathrm{g}} \Delta i_{\mathrm{c}} .
\end{aligned}
$$

The species conservation equation is the same as that in the two-medium treatment given by equation (21). The species diffusion coefficient is obtained by assuming a unity effective Lewis number for the singlemedium, i.e.

$$
L e_{\mathrm{e}_{1}}=\frac{\frac{\left\langle k_{\mathrm{e}}\right\rangle}{\left(\rho c_{\mathrm{p}}\right)_{\mathrm{g}}}+D_{x x}^{\mathrm{d}}}{\left\langle D_{\mathrm{e}}\right\rangle+D_{\mathrm{m}_{x x}}^{\mathrm{d}}}=1 .
$$

For the effective conductivity $\left\langle k_{\mathrm{e}}\right\rangle$, we use the exact results of Sahraoui and Kaviany [11], i.e.

$$
\begin{aligned}
& \frac{\left\langle k_{\mathrm{e}}\right\rangle}{k_{\mathrm{g}}}= \\
& \frac{f(\varepsilon) \frac{k_{\mathrm{s}}}{k_{\mathrm{g}}}(1-\varepsilon)^{1 / 2}+\left[1-(1-\varepsilon)^{1 / 2}\right]+[1-f(\varepsilon)](1-\varepsilon)^{1 / 2}}{\frac{k_{\mathrm{s}}}{k_{\mathrm{g}}}(1-\varepsilon)^{1 / 2}\left[1-(1-\varepsilon)^{1 / 2}\right]+\left[1-(1-\varepsilon)^{1 / 2}\right]^{2}+(1-\varepsilon)^{1 / 2}}
\end{aligned}
$$

wherc $f(\varepsilon)$ is introduced to account for the two-dimensional effects, and for the square cylinders is given by

$$
f(\varepsilon)=0.83+0.18(1-\varepsilon) .
$$


For the continuous solid phase, the total stagnant effective conductivity is obtained by adding equations (24) and (25). The inlet boundary conditions are given by

$$
\langle T\rangle=T_{\mathrm{n}} \quad\langle Y\rangle^{\mathrm{g}}=0 \quad \text { for } \quad x \rightarrow-\infty
$$

and the exit boundary conditions are given by

$$
\frac{\partial\langle T\rangle}{\partial x}=\frac{\partial\langle Y\rangle^{\mathrm{g}}}{\partial x}=0 \text { for } \quad x \rightarrow \infty
$$

\section{RESULTS AND DISCUSSION}

In this section, after discussing some typical results, the computed area-average Nusselt number for the staggered and in-line arrangements are presented. These results are used in the two-medium treatment as a piecewise linear correlation giving the Nusselt number as a function of the Reynolds number. Then the results of the two-dimensional simulations and the volume-averaged models are presented first for the discrete and then for the continuous solid phase porous media. In addition to the axial temperature distribution and the flame speed, the local increase in the temperature above the adiabatic temperature is also discussed. The dimensionless excess temperature is defined as

$$
\Delta T_{\max }^{*}=\frac{T_{\max }-T_{\mathrm{a}}}{T_{\mathrm{a}}-T_{\mathrm{n}}} .
$$

The effects of the flame location $x_{\mathrm{F}}$, porosity $\varepsilon$, conductivity ratio $k_{\mathrm{s}} / k_{\mathrm{g}}$, particle arrangement, pore size $l$, and equivalence ratio $\Phi$ on the flame speed are examined for the discrete solid phase. For the continuous solid phase, the effects of $x_{\mathrm{F}}$ and $k_{\mathrm{s}} / k_{\mathrm{g}}$ are examined. These parameters are also included in the volume-averaged models (except for the flame location within the pore, since these models mask this pore-level variation).

\subsection{Typical results}

Typical steady-state results for the temperature distribution, obtained using the two-dimensional simulation and the one-dimensional volume-averaged models, are shown in Fig. 2. The two-dimensional results are for the in-line arrangement of discrete square cylinders with $\varepsilon=0.9, k_{\mathrm{s}} / k_{\mathrm{g}}=100, l=1 \mathrm{~mm}$, and $\Phi=1$. The two-dimensional result shows a curvature in the flame front due to the presence of the high-conductivity solid. The location of the maximum rate of reaction, i.e. the flame location $x_{F}$, occurs above the solid particle, and the particle temperature is uniform because $k_{\mathrm{s}} / k_{\mathrm{g}}$ is rather high. This also causes some upstream heat diffusion, resulting in the enhanced preheat of the reactants. This preheat, in addition to increasing the flame speed, causes the local temperature in the gas to increase above the adiabatic temperature. This is shown in Fig. 2(a), in the region where the local temperatures are higher than $2400 \mathrm{~K}$. The highest temperature occurs at $y / l=0.5$ and is about $2560 \mathrm{~K}$. The adiabatic flame temperature $T_{\mathrm{a}}$ for this methane-air system with an equivalence ratio of unity, is $2308 \mathrm{~K}$, and, therefore, the maximum temperature difference $T_{\mathrm{g}}-T_{\mathrm{a}}$ is $252 \mathrm{~K}$. In the post-flame region, a redistribution of heat occurs and further downstream a uniform temperature is found. For this case the flame specd is about $5 \%$ smaller than that of the adiabatic flame speed in plain media $u_{\mathrm{F}}$, i.e. $u_{\mathrm{F}} / u_{\mathrm{F}_{\mathrm{u}}}=0.95$. Figure 2(b) shows the one-dimensional temperature distributions in the solid and gas effective media obtained from the two-medium, local volumeaveraged model. The thermal nonequilibrium between the two phases is apparent. In the reaction zone the gas has a higher temperature, compared to the solid, causing heat transfer from the effective gas medium to the effective solid medium. Heat is conducted through the solid medium to the upstream region where the solid medium temperature is higher than the gas medium, and this preheats the gas medium before it arrives in the reaction zone. This enhanced (over the amount occurring through the gas medium) preheat results in an increase in the flame speed and the occurrence of the excess temperature (over the adiabatic temperature). For the two-medium treatment, the maximum temperature reached by the gas medium is $2351 \mathrm{~K}$, resulting in an excess temperature of $43 \mathrm{~K}$ [this cannot be distinguished in Fig. 2(b)]. Figure 2(c) shows the temperature distribution predicted by the single-medium treatment which assumes a local thermal equilibrium between the gas and solid phases. In the single-medium treatment no excess temperature occurs. From Fig. 2(b) and (c), we notice that the volume-averaged models predict the flame thickness fairly well.

Similarly, typical steady-state results for the temperature distribution for the connected solid phase are shown in Fig. 3 for the two-dimensional simulation and the volume-averaged modcls. The solutions are also for the in-line arrangement of cylinders with $\varepsilon=0.9, k_{\mathrm{s}} / k_{\mathrm{g}}=100, l=1 \mathrm{~mm}, \Phi=1$, and $c / d=0.1$. Figure 3(a) shows the temperature distribution for the two-dimensional simulation. The connecting arms between adjacent square cylinders are assumed to be very permeable (i.e. no resistance to fluid flow) and have the same conductivity as that of the solid. Due to the presence of the connecting arms, the flame thickness and the preheat are further enhanced, causing a $60 \%$ increase in the flame speed compared to the speed in plain media, i.e. $u_{\mathrm{F}} / u_{\mathrm{F}}=1.6$. The maximum local temperature occurs at $y / l=0.5$ and is about $2680 \mathrm{~K}$, i.e. an excess temperature of $362 \mathrm{~K}$. The results for the two-medium treatment are shown in Fig. 3(b). As observed in the two-dimensional solution, the two-medium model also shows an increase in the extent of the thermal nonequilibrium, i.e. a higher preheat and an increase in the flame speed. The two-medium treatment predicts the flame thickness fairly well. The results from the single-medium treatment are shown in Fig. 3(c), and a smaller flame thickness and no excess temperature are predicted. 


\subsection{Nusselt number}

The computed results of the area-averaged Nusselt number $\left\langle N u_{1}\right\rangle_{A_{\mathrm{gs}}}$ are shown in Table 2 for the in-line and staggered arrangements of square cylinders. The variation of $\left\langle N u_{1}\right\rangle_{A_{g s}}$ between the square particles is not noticeable (except for the first and last cylinders in the computational domain). The results for $\left\langle N u_{1}\right\rangle_{A_{\mathrm{gs}}}$ are reported for the range of Reynolds number used in this study. For both particle arrangements, the Nusselt number decreases with $R e_{1}$, for $R e_{1}<20$. This is due to the decrease in the local Nusselt number on the upstream side of the particle, as shown in the polar plot of the computed local Nusselt number $N u_{1}$ in Fig. 4(a). For the in-line arrangement of the square cylinders, as $R e_{1}$ increases the flow becomes more rectilinear and the heat removal from the upstream side decreases. At higher $R e_{1}$ (i.e. $R e_{1}>20$ ), $N u_{1}$ increases slightly at the top surface (along the flow), and, therefore, $\left\langle N u_{1}\right\rangle_{A_{\mathrm{gs}}}$ is not strongly affected by the variation in $R e_{1}$. For the staggered arrangement, the fluid follows a more tortuous path and the change in $\left\langle N u_{1}\right\rangle_{A_{\mathrm{gs}}}$ with respect to $R e_{1}$ is more pronounced. Figure 4(b) shows that, as $R e_{1}$ increases, $N u_{1}$ increases at the top and upstream surfaces. The increase in the heat transfer on the upstream surface is due to the stagnation point flow behavior as the flow inertia increases.

\subsection{Discrete solid phase}

The effects of the various parameters on the flame structure and specd are now examined for the discretc solid phase.

4.3.1. Effect of flame location. The effect of the flame location is examined for the in-line arrangement using $k_{\mathrm{s}} / k_{\mathrm{g}}=1$. This allows the examination of the effect of the pore-level hydrodynamics on the flame speed and the excess temperature (because the effect of the solidphase conduction is not very significant). The variation of the normalized flame speed (again $u_{\mathrm{F}_{0}}$ is the adiabatic flame speed in plain media) with respect to the location of the flame is shown in Fig. 5 and the results show that, as the fiame is displaced from the region above the cylinder (i.e. $x_{\mathrm{F}} / l=0$ ), to the region between the cylinders (i.e. $x_{\mathrm{F}} / l=0.5$ ), the flame speed increases. This is because, when the flame is away from the cylinder, the flow converges in the wake region and causes a uniform temperature in the reaction zone, resulting in a higher temperature in the wake region. The gas and cylinder conduct the heat upstream and preheat the nonreacted mixture. The conduction in the wake region is significant because the local Péclet number is small. For the case of $x_{\mathrm{F}} / l=0.5$, the nonuniformity of the temperature in the preheat zone is more pronounced than that for $x_{F} / l=0$, which results in more preheat and consequently higher flame speeds. The variation of the dimensionless excess temperature $\Delta T_{\max }^{*}$ with the flame location shows that, for $x_{\mathrm{F}} / l=0, \Delta T_{\max }^{*}$ is 0.071 which is higher than $\Delta T_{\max }^{*}=0.036$ found for $x_{\mathrm{F}} / l=0.5$. This is because, for the latter, the tem- perature distribution is more uniform in the reaction zone. Figure 5 also shows the results of the single- and two-medium treatments. A constant value is shown because these models do not account for the porelevel variations. The two-medium treatment predicts a flame speed that is higher than the two-dimensional flame speed, for all flame locations.

4.3.2. Effect of porosity. The effect of the porelevel hydrodynamics is also examined by varying the porosity and Fig. 6 shows the variations of the flame speed with respect to the flame position for $\varepsilon=0.8$ and 0.9 and $k_{\mathrm{s}} / k_{\mathrm{g}}=1$. For all flame locations, the flame speed for $\varepsilon=0.8$ is lower than that for $\varepsilon=0.9$. This is mainly a hydrodynamic effect, because the local velocity within the pore (or gas phase averaged velocity) increases as $\varepsilon$ decreases. Examination of the local temperature shows that the preheat is larger for $\varepsilon=0.8$ and this should result in an increase in the flame speed. However, the effect of the increase in the local velocity within the pore dominates and a lower flame speed is obtained. The effect of the increase in the preheat for $\varepsilon=0.8$ causes an increase in the excess temperature as shown in Table 3. The effect of porosity is also studied for $k_{\mathrm{s}} / k_{\mathrm{g}}=100$ and it is found that the flame speed increases with the decrease in porosity. This is due to the presence of a greater highconductivity solid volume within the flame.

The effect of porosity is also studied using the singleand two-medium treatments, and the trends are different from that for the two-dimensional flames. The rcsults are shown in Table 3, where the volumeaveraged models show an increase in the flame speed with the decrease in porosity. This increase in the flame speed, with a decrease in porosity, is due to the increase in the effective conductivity of the solid medium, which increases the preheat. Also, the hydrodynamic dispersion coefficient is larger for $\varepsilon=0.8$ and this results in an increase in the flame speed. Note that, as the porosity decreases, the source term $\varepsilon\langle\dot{n}\rangle^{g}$ in equation (21) tends to decrease the flame speed. However, the increase in the solid medium effective conductivity and the hydrodynamic dispersion more than compensate for this decrease. An increase in the solid medium effective conductivity also results in an increase in the dimensionless excess temperature, as shown in Table 3. These results show that the volumeaveraged models do not predict the magnitude of the flame speed accurately, and also do not predict some of the trends such as the variation of $u_{\mathrm{F}}$ with $\varepsilon$. This is mainly due to the errors introduced in the prescribed effective conductivity and the axial hydrodynamic dispersion coefficient, and also due to the source term which assumes a completely mixed pore.

4.3.3. Effect of $\mathrm{k}_{s} / \mathrm{k}_{q}$. As was mentioned, one advantage of using a porous medium is the high conductivity of the solid phase, which enhances the preheating of the reactant. Figure 7 shows that, when the flame is located above the cylinder, the flame speed is greatly affected by the solid-phase conductivity. For large $k_{\mathrm{s}} / k_{\mathrm{g}}$, the preheat is substantial, as shown in Fig. 8(a) 
(a) Two-Dimensional Simulation
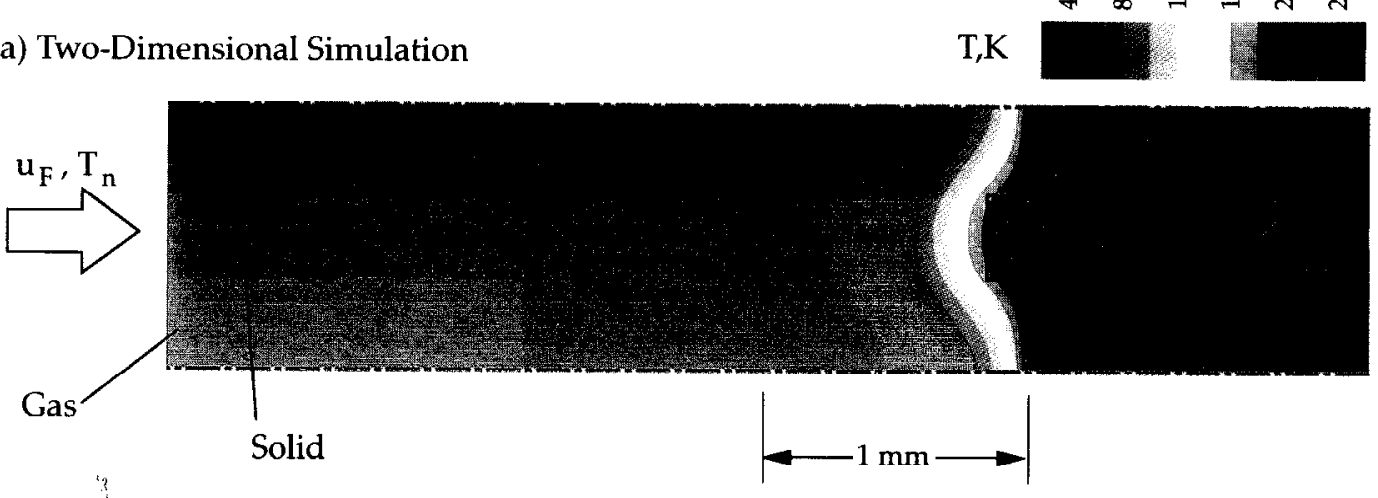

(b) Two-Medium Volume Averaged

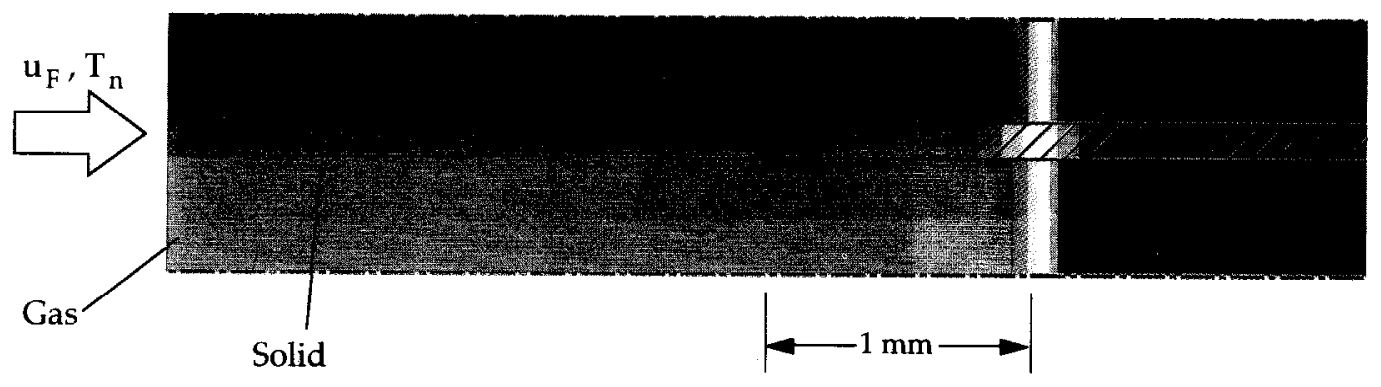

(c) Single-Medium Volume Averaged

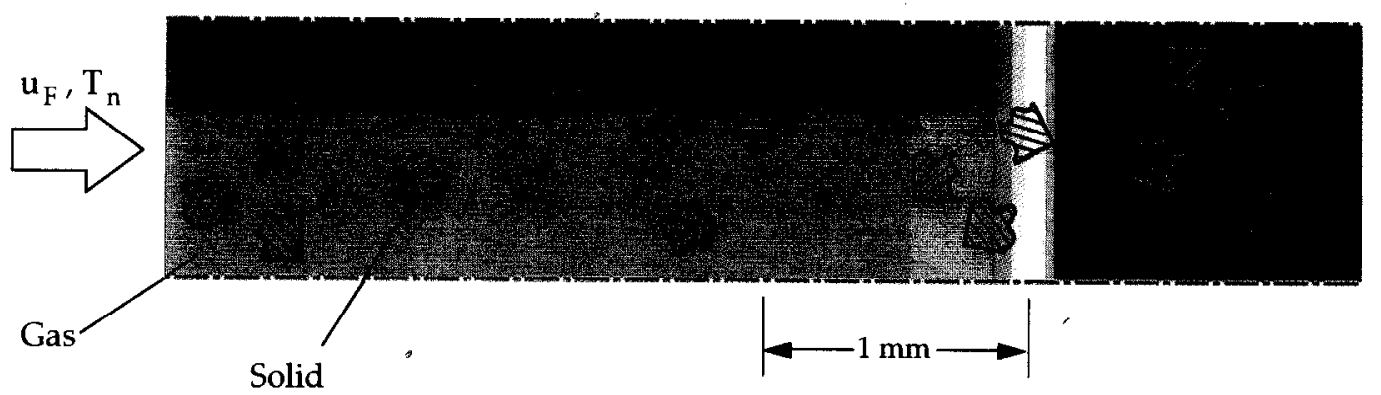

Fig. 2. Temperature distribution for adiabatic, premixed flame using the in-line arrangement of discrete square cylinders. The results are for $\varepsilon=0.9, k_{\mathrm{s}} / k_{\mathrm{g}}=100, \Phi=1$, and different models : (a) two-dimensional; (b) one-dimensional, two-medium; and (c) one-dimensional, single-medium. 


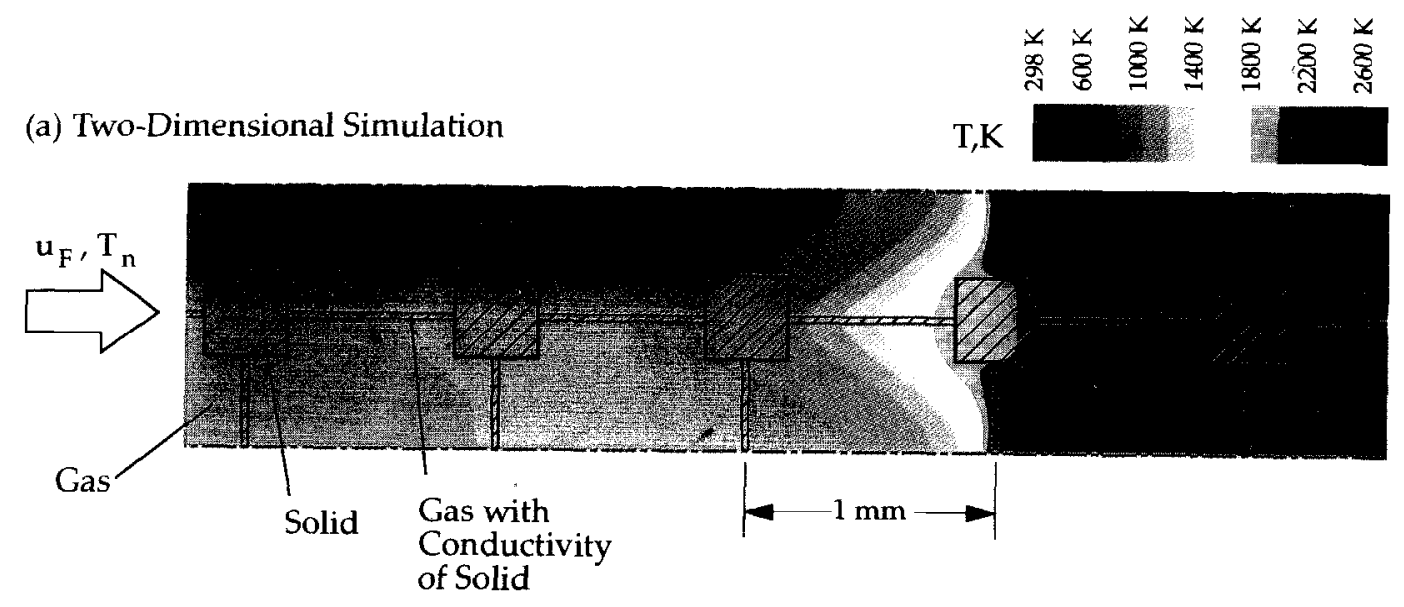

(b) Two-Medium Volume Averaged

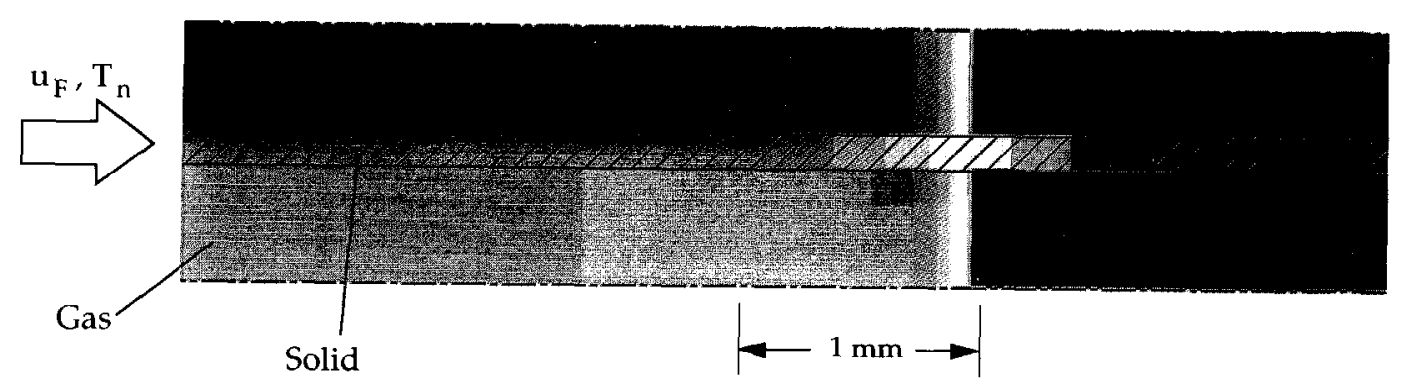

(c) Single-Medium Volume Averaged

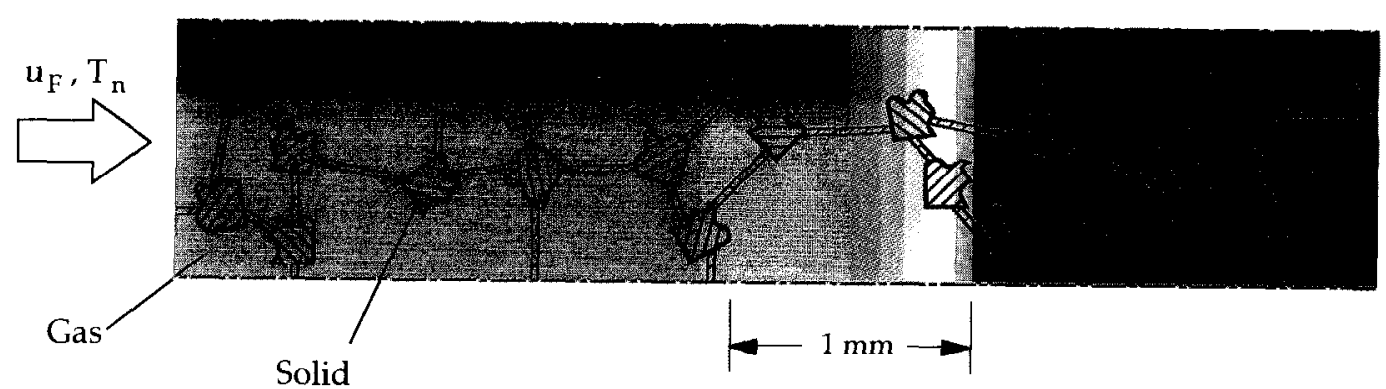

Fig. 3. Same as Fig. 2, but for a continuous solid phase $(c / d=0.1)$. 
Table 2. Variation of the area-averaged Nusselt number with respect to the Reynolds number for the in-line and staggered arrangements of square cylinders, $P r=0.67$

\begin{tabular}{lccc}
\hline & \multicolumn{3}{c}{$\left\langle N u_{1}\right\rangle_{\mathbf{s}_{\mathrm{ss}}}$} \\
\cline { 2 - 3 } & \multicolumn{2}{c}{ In-line } & Staggered \\
${$\cline { 2 - 3 }$} }$ & $\varepsilon=0.9$ & $\varepsilon=0.8$ & $\varepsilon=0.9$ \\
\hline 0.23 & 7.62 & 7.45 & 8.77 \\
2.28 & 7.12 & 6.90 & 8.20 \\
4.55 & 6.85 & 6.62 & 7.97 \\
9.10 & 6.64 & 6.41 & 8.01 \\
22.8 & 6.53 & 6.30 & 8.96 \\
45.5 & 6.57 & 6.39 & 10.6 \\
91.0 & 6.81 & 6.52 & 12.8 \\
\hline
\end{tabular}

for $x_{\mathrm{F}} / l=0$ and $k_{\mathrm{s}} / k_{\mathrm{g}}=100$, where the local temperature difference between the solid and gas at two lateral locations, $y / l=0$ (passing through the solid) and $y / l-0.5$ (not passing through the solid), is significant due to the conduction through the solid phase. For large $k_{\mathrm{s}} / k_{\mathrm{g}}$, the flame speed approaches an asymptotic value which depends on the flame location. This is because the cylinders are not connected, and, as

(a) In-Line

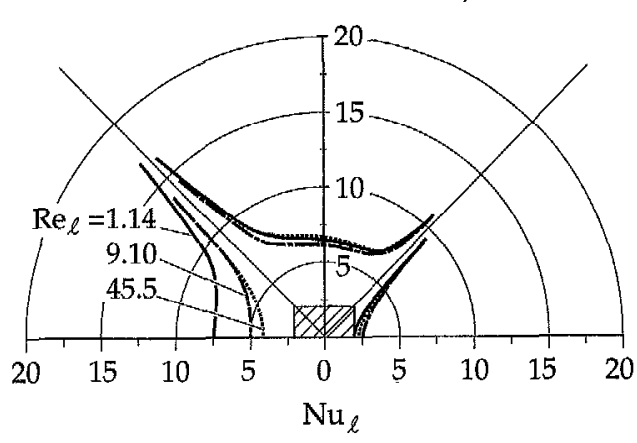

(b) Staggered

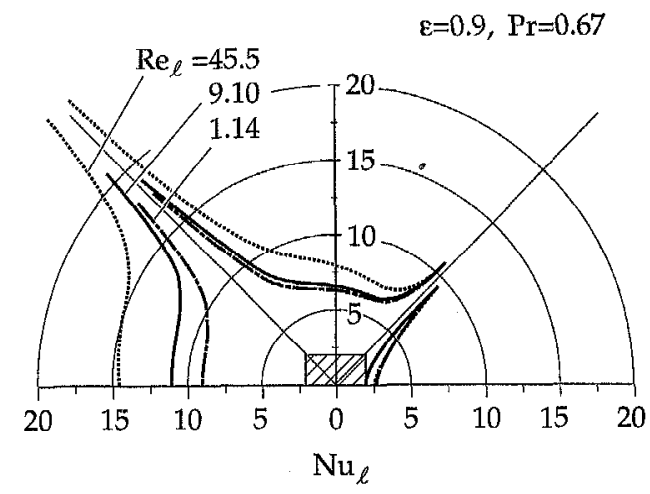

Fig. 4. Polar representation of the local Nusselt number distribution on the surface of a square cylinder for: (a) inline arrangement, and (b) for staggered arrangement.

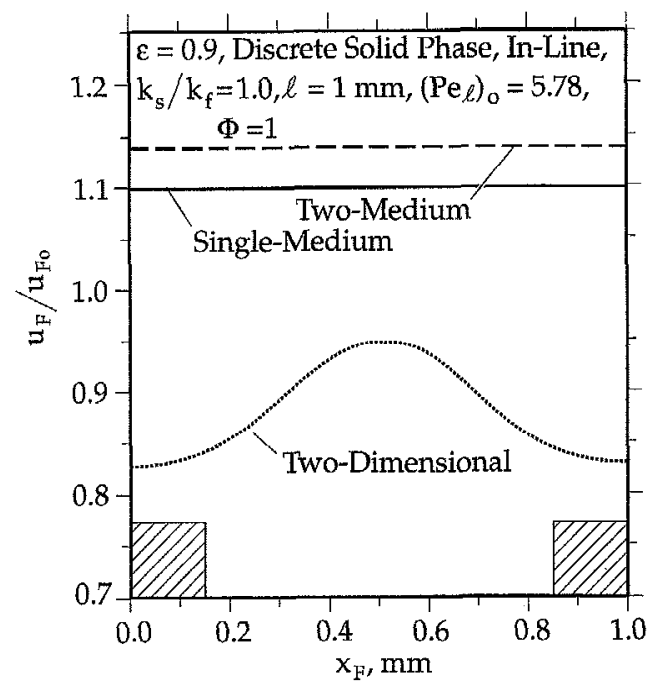

Fig. 5. Variation of the normalized flame speed with the flame location as obtained from the two-dimensional solutions and the volume-averaged models.

shown by Sahraoui and Kaviany [11], the effective conductivity reaches an asymptote value as $k_{\mathrm{s}} / k_{\mathrm{g}}$ becomes very large. When the flame is located away from the cylinder (i.e. $x_{\mathrm{F}} / l=0.5$ ), the effect of the solid conductivity is not significant and the flame velocity is only affected by the local velocity gradient which reduces the flame speed compared to the one-dimensional adiabatic flame speed (in plain media). The local temperature distributions for $x_{\mathrm{F}} / l=0.5$ at $y / l=0$ and 0.5 are shown in Fig. $8(\mathrm{~b})$, where it is found that in the $y$-direction the temperature in more uniform compared to that for $x_{\mathrm{F}} / l=0$. The $y$-direction nonuniformity existing at the beginning of the preheat zone does not affect the flame speed significantly. The distribution of the local product mass

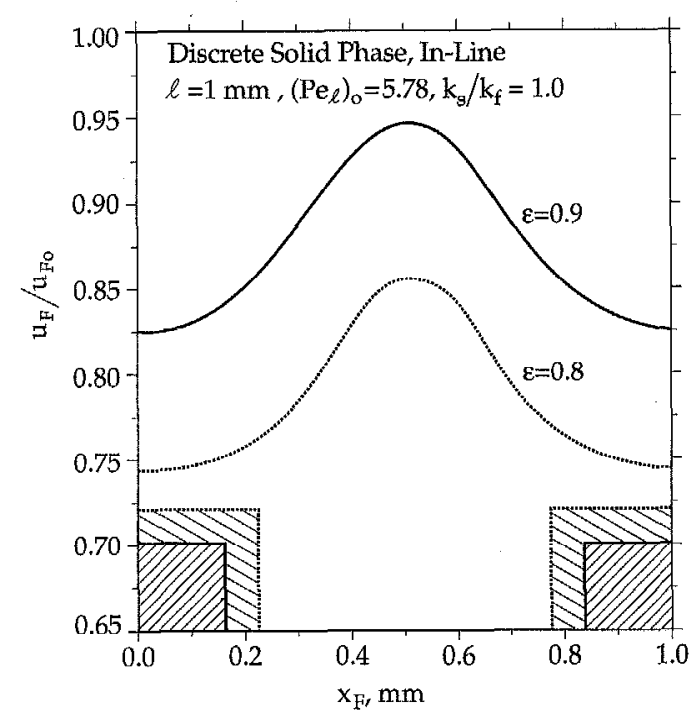

Fig. 6. Variation of the normalized flame speed with the flame location for two different porosities. 
fraction is shown in Fig. 9(a) and (b), for $x_{\mathrm{F}} / l=0$ and 0.5 . These results show that, for both cases, the reaction is initiated in the wake region of the cylinder, where the effect of the preheat is more significant.

Figures 8 and 9 also show the predicted temperature and mass fraction obtained from the single-and twomedium treatments. These results show that the predicted volume-averaged mass fraction and temperature are within the range of the local distributions predicted from the two-dimensional solution (for both flame positions). The two-medium model predicts an excess temperature which is lower than the maximum temperature found from the two-dimensional solution. The single-medium model does not predict an excess temperature. Therefore, the two-medium model can predict the gross features (i.e. the flame speed, axial temperature, axial mass fraction, and flame thickness) of the flame, but not the pore-level features (such as the variation of the flame speed with respect to the position and the local high temperatures within the pores).

4.3.4. Effect of pore size. So far, the effect of $k_{\mathrm{s}} / k_{\mathrm{g}}$ and $x_{\mathrm{F}} / l$ on the flame structure has been examined for a flame thickness smaller than the unit-cell size $l$. The local volume-averaging theory does not strictly apply to this case. Now consider a smaller pore size such that, while keeping the pressure and the equivalence ratio the same, the flame spread over more than a square cylinder. We consider $l=0.5 \mathrm{~mm}$ and the results for the variation of the flame speed with respect to $k_{\mathrm{s}} / k_{\mathrm{g}}$ are presented in Fig. 10, for the unit-cell sizes $l=0.5$ and $1 \mathrm{~mm}$, and for two flame positions. The results show that the flame speed for the smaller unitcell size is larger (for both flame positions, but more pronounced for $x_{\mathrm{F}} / l=0$ ). For $l=0.5 \mathrm{~mm}$ the flame spreads over a few cylinders, resulting in a larger preheat and an increase in the flame speed. For small

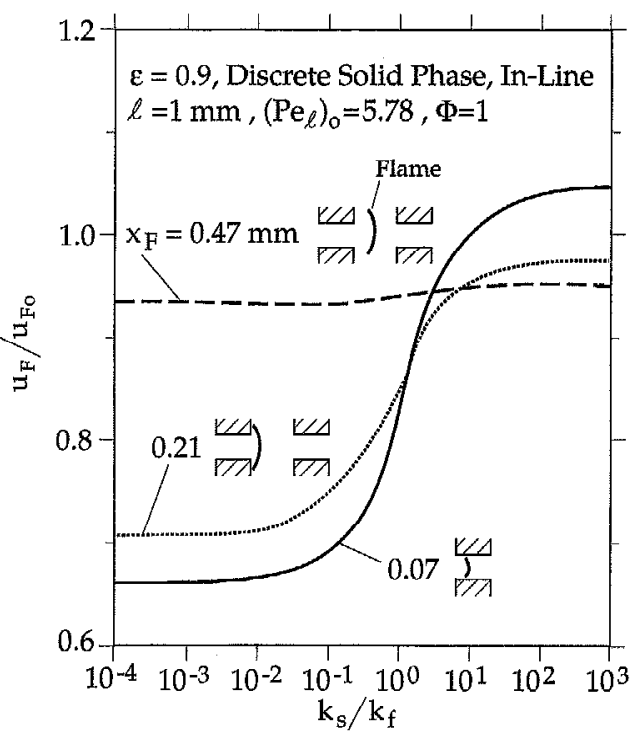

Fig. 7. Effect of $k_{\mathrm{s}} / k_{\mathrm{g}}$ on the normalized flame speed for three different flame locations within the pore. (a)
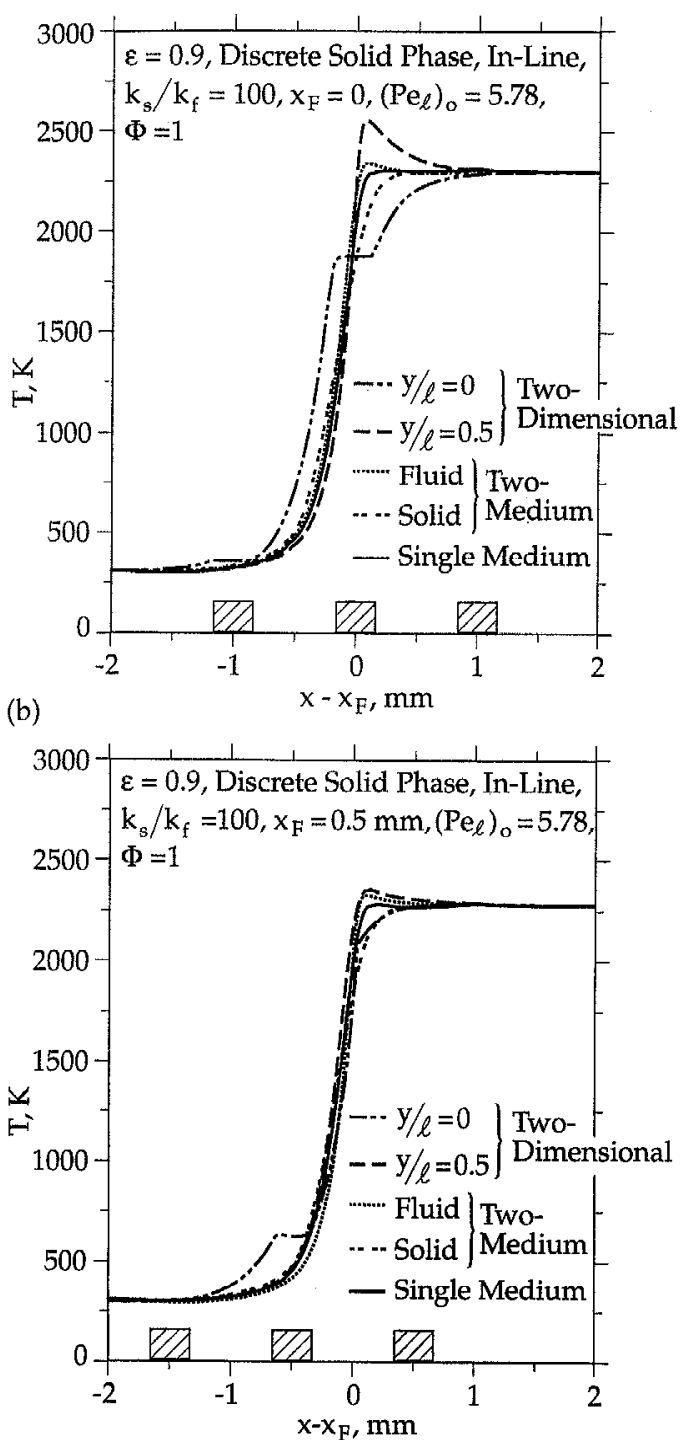

Fig. 8. Axial temperature distribution at two lateral positions obtained from the two-dimensional solutions and compared with those from the single- and two-medium models: (a) $x_{\mathrm{F}} / l=0$, and (b) $x_{\mathrm{F}} / l=0.5$.

$k_{\mathrm{s}} / k_{\mathrm{g}}$ and $x_{\mathrm{F}} / l=0.5$, the flame speed is smaller for $l=0.5 \mathrm{~mm}$, and this is due to the hydrodynamic effects since the flame thickness is about one unit-cell size and more solid surface areas are present within the flame. For $x_{\mathrm{F}} / l=0.5$ the flame speed does not change significantly with the variation in $k_{\mathrm{s}} / k_{\mathrm{g}}$ because the solid is away from the high-temperature region, and, therefore, the preheat is reduced. The results for the flame speed and the dimensionless excess temperature along with the prediction of the volumeaveraged models are shown in Table 4 , for $k_{\mathrm{s}} / k_{\mathrm{g}}=100$. For the two flame locations in the two-dimensional simulations, the excess temperature decreases with the decrease in the unit-cell size. This is because, as the unit-cell size decreases, the local Péclet number 
(a)

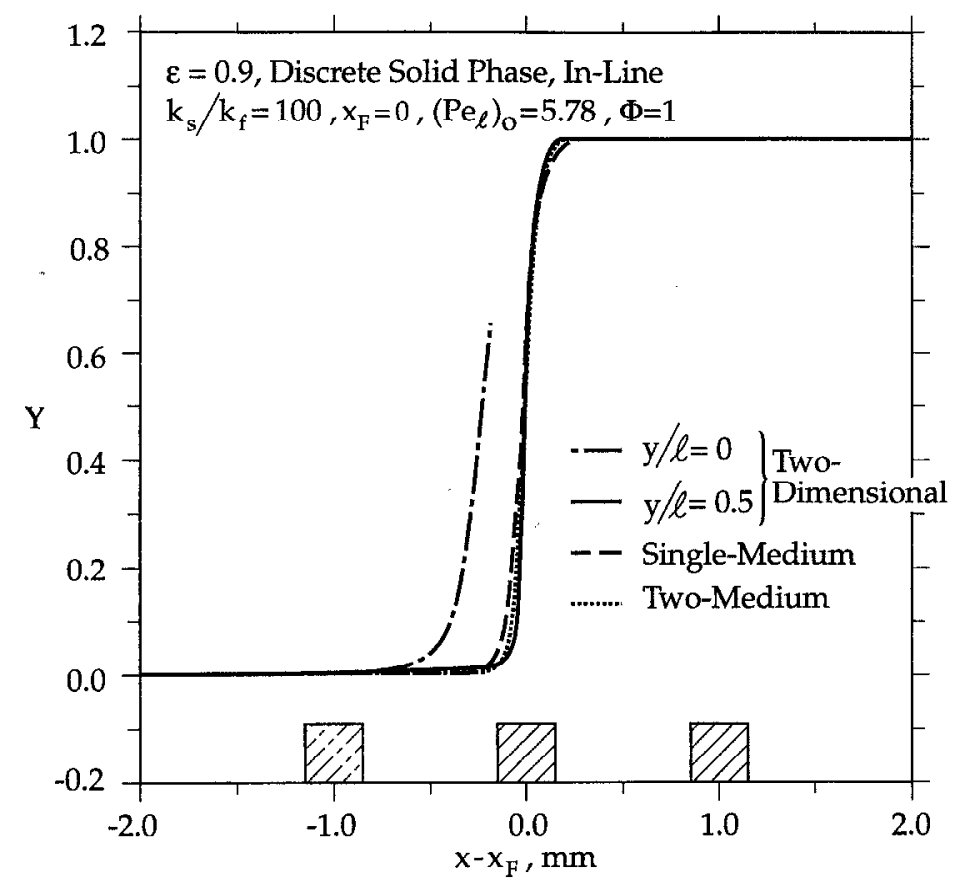

(b)

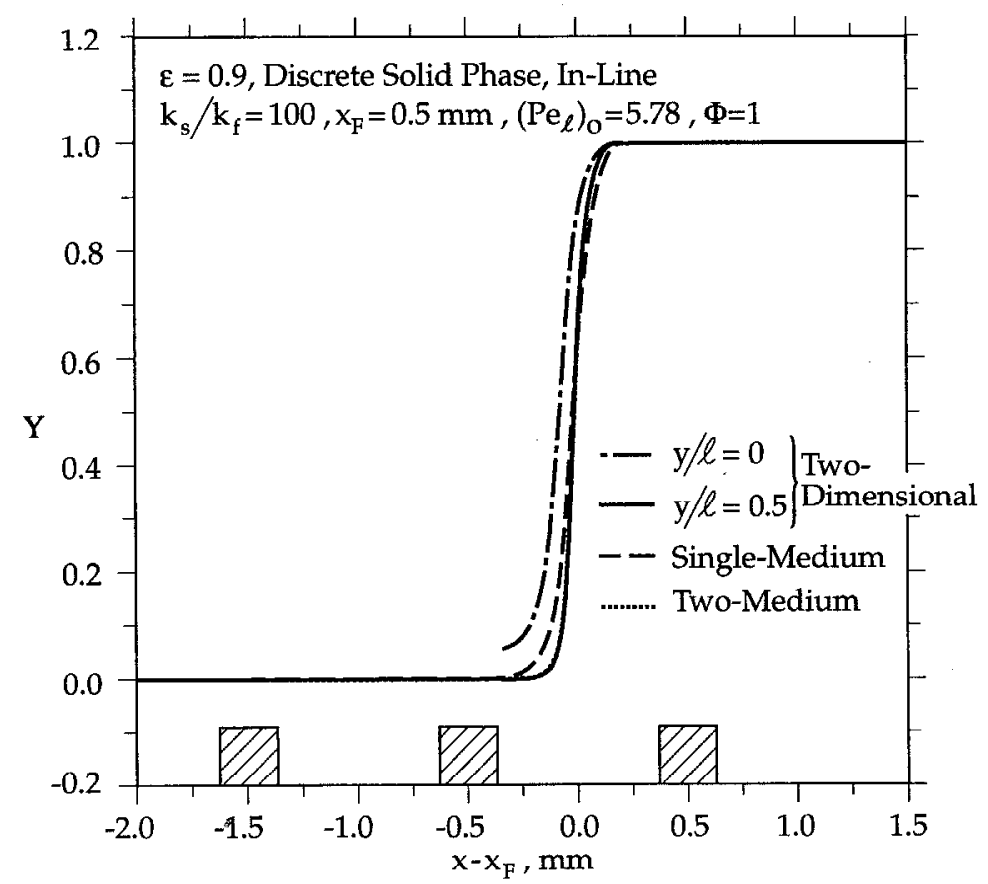

Fig. 9. Mass fraction distribution at two lateral positions obtained from the two-dimensional solutions and compared with those from the single- and two-medium models for: (a) $x_{\mathrm{F}} / l=0$, and (b) $x_{\mathrm{F}} / l=0.5$.

decreases and the heat diffusion becomes more significant. This decreases the temperature nonuniformity within the pore.

The two-dimensional results for flame speed for the two unit-cell sizes are compared with the results predicted from the volume-averaged models, as shown in Table 4. The two-dimensional model predicts an increase in the flame speed as $l$ decreases. However, the volume-averaged models predict a decrease in the flame speed as $l$ decreases. This is because, as $l$ decreases, the gas Péclet number decreases and the hydrodynamic dispersion coefficient decreases, result- 
Table 3. Effect of porosity variation on the flame speed and the dimensionless excess temperature, in-line arrangement and $k_{\mathrm{s}} / k_{\mathrm{g}}=1$

\begin{tabular}{ccccc}
\hline & \multicolumn{3}{c}{$u_{\mathrm{F}} / u_{\mathrm{F}_{\mathrm{u}}}\left(\Delta T_{\max }^{*}\right)$} \\
\cline { 2 - 3 }$\varepsilon$ & \multicolumn{2}{c}{ Two-dimensional } & \\
\cline { 2 - 3 } 0.8 & $x_{\mathrm{F}} / l=0$ & $x_{\mathrm{F}} / l=0.5$ & Two-medium & Single-medium \\
& 0.74 & 0.85 & 1.18 & 1.12 \\
0.9 & $(0.091)$ & $(0.055)$ & $(0.015)$ & 1.10 \\
& 0.83 & 0.95 & 1.14 & \\
& $(0.071)$ & $(0.036)$ & $(0.013)$ & \\
\hline
\end{tabular}

ing in a smaller flame specd. By taking out the effect of dispersion in the two-medium treatment (i.e. $D_{x x}^{\mathrm{cl}}=0$ ), we found that the flame speed increases as $l$ decreases (as predicted from the two-dimensional model). This is due to the increase in the heat transfer between the two phases.

4.3.5. Particle arrangement. The pore-level hydrodynamic effect influences the flame structure and speed, and this was shown in Fig. 5, where $x_{\mathrm{F}}$ was varied in the pore for $k_{\mathrm{s}} / k_{\mathrm{g}}=1$. Here, the hydrodynamic effect is further examined by changing the arrangement of the square cylinders. The flame speed is examined for the staggered arrangement of square cylinders shown in Fig. 1(b). For this arrangement, the fluid path is tortuous, thus altering the flame structure and resulting in a higher flame speed compared to that for the in-line arrangement, as shown in Fig. 11. The increase in the flame speed is especially significant for $x_{\mathrm{F}} / l=0.5$, where the flame is located in the wake of the cylinder. In this region and for the staggered arrangement, the flow is more uniform compared to the in-line arrangement. The uniformity of the flow leads to a flame structure approaching that for the one-dimensional flame. For $x_{\mathrm{F}} / l=0$, the flame

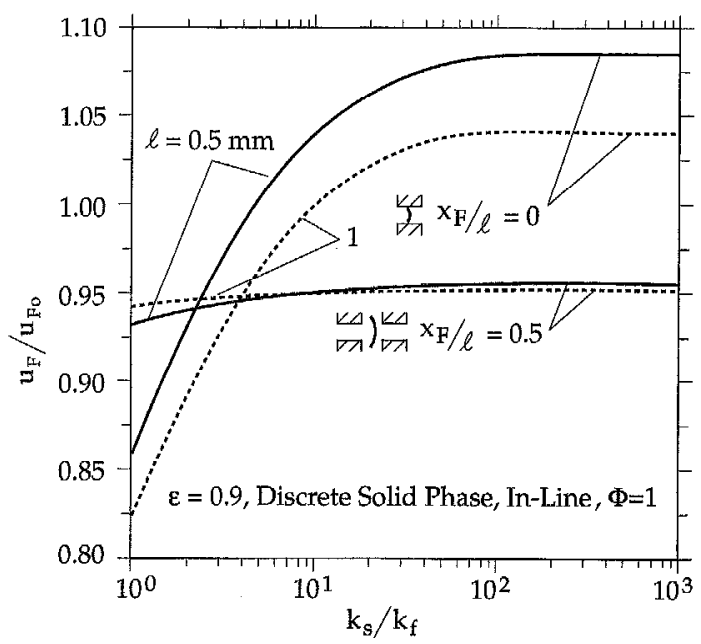

Fig. 10. Effect of the unit-cell size $l$ on the normalized flame speed for variable $k_{\mathrm{s}} / k_{\mathrm{g}}$ and for two different flame positions within the pore. speed is also higher for the staggered arrangement, due to the higher temperature of the cylinder in the reaction zone. As a result, the preheating and the flame speed also increase. The excess temperature is also increased as shown in Table 5 . The higher cylinder temperature is due to the transverse components of the velocity which is more significant in the staggered arrangement.

In Table 5, the results from the two-dimensional solutions are compared to those from the volumeaveraged models. The volume-averaged predictions show a good agreement with the two-dimensional results, when the flame is located at $x_{\mathrm{F}} / l=0$. However, the agreement is less when $x_{\mathrm{F}} / l=0.5$ for which a difference of $11 \%$ exists. For both arrangements, the flame speed predicted by the single- and two-medium treatments are very close. The volumeaveraged models predict a lower flame speed, because the hydrodynamic dispersion coefficient is smaller for the staggered arrangement.

4.3.6. Equivalence ratio. As the equivalence ratio $\Phi$ varies, the flame structure within the pore changes. The flame thickness increases, and the flame speed and the product temperature decrease, with a decrease

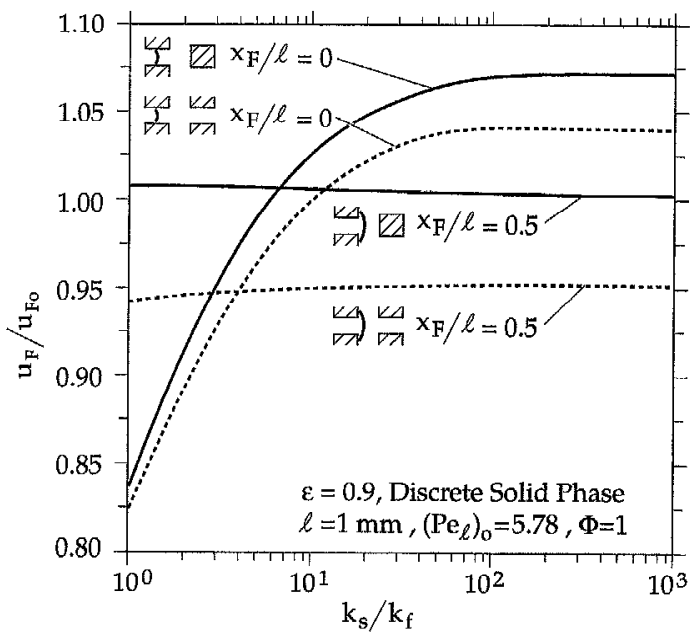

Fig. 11. Effect of the particle arrangement on the normalized flame speed, for the staggered and in-line arrangements of square cylinders, for variable $k_{\mathrm{s}} / k_{\mathrm{g}}$ and for two different flame positions within the pore. 
Table 4. Effect of cell size $l$ on the flame speed and the dimensionless excess temperature, in-line arrangement, $k_{\mathrm{s}} / k_{\mathrm{g}}=100$, and $\varepsilon=0.9$

\begin{tabular}{ccccc}
\hline & \multicolumn{3}{c}{$u_{\mathrm{F}} / u_{\mathrm{F}_{\mathrm{o}}}\left(\Delta T_{\max }^{*}\right)$} \\
\cline { 2 - 3 }$l$ & \multicolumn{2}{c}{ Two-dimensional } & & \\
\cline { 2 - 3 }$[\mathrm{mm}]$ & $x_{\mathrm{F}} / l=0$ & $x_{\mathrm{F}} / l=0.5$ & Two-medium & Single-medium \\
\hline 0.5 & 1.08 & 0.96 & 1.16 & 1.12 \\
& $(0.063)$ & $(0.021)$ & $(0.014)$ & 1.21 \\
& 1.04 & 0.95 & 1.22 & \\
\hline
\end{tabular}

in the equivalence ratio. Figure 12 shows that, as with the decrease in the cell size, $u_{\Gamma} / u_{\Gamma}$ increases as $\Phi$ decreases. As $\Phi$ decreases and the flame thickness increases, more solid particles are present within the flame region, and, therefore, the amount of preheat is increased. A comparison with the results of a decrease in $l$, shown in Fig. 10, shows that the increase in $u_{\mathrm{F}} / u_{\mathrm{F}_{\mathrm{o}}}$ is larger when $\Phi$ changes, because the ratio of the flame thickness to the unit-cell size is larger.

The flame speed and the dimensionless excess temperature predicted from the two-dimensional solutions are also compared with those obtained for the volume-averaged models, and are shown in Table 6. The two-dimensional results for $u_{\mathrm{F}} / u_{\mathrm{F}_{\mathrm{o}}}$ are larger when the cylinder is located in the reaction zone (i.e. $x_{F} / l=0$ ), because of the larger solid temperature and the increase in the preheat. In the two-medium treatment, for $\Phi=0.7$ the predicted flame speed is very close to the two-dimensional flame speed for $x_{\mathrm{F}} / l=0$. For $\Phi=0.7$ the dimensionless excess temperature is smaller, because the flame thickness is larger and the lateral nonuniformity in the temperature is reduced.

\subsection{Continuous solid phase}

The results presented above show that the effect of conduction through the solid is significant and influ-

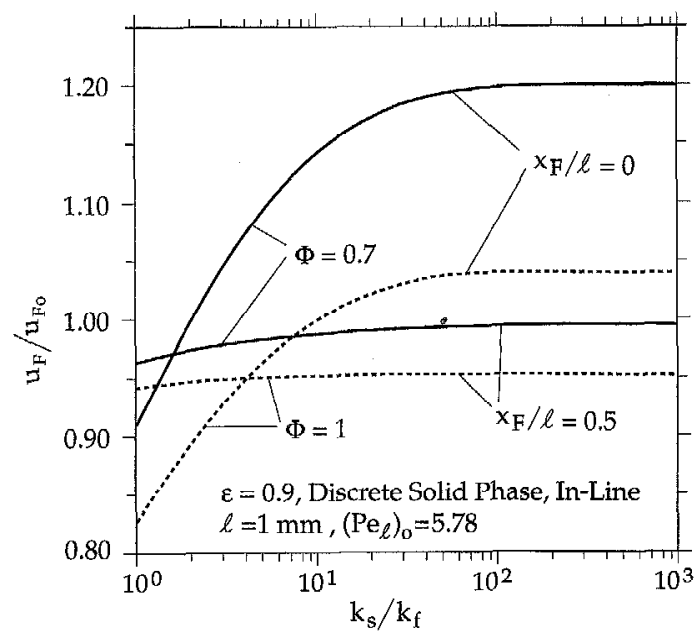

Fig. 12. Effect of the equivalence ratio on the normalized flame speed for variable $k_{\mathrm{s}} / k_{\mathrm{g}}$ and for the in-line arrangement of square cylinders. ences the flame structure and speed in the porous medium. This becomes even more significant when the cylinders are connected, and there the effective conductivity of the medium is not bounded as $k_{\mathrm{s}} / k_{\mathrm{g}}$ is increased. For the case of interconnected cylinders as shown in Fig. 1(c), with $c / d=0.1$ and $k_{\mathrm{s}} / k_{\mathrm{g}}=100$, the local temperature distributions at $y / l=0$ and 0.5 are shown in Fig. 13. Also shown in Fig. 13, are the results of the volume-averaged models. The two-dimensional solution predicts a maximum temperature of $2947 \mathrm{~K}$ (i.e. $\Delta T_{\max }^{*}=0.32$ ) occurring at $y / l=0.5$. The twomedium model predicts a maximum temperature of $2522 \mathrm{~K}$ (i.e. $\Delta T_{\max }^{*}=0.11$ ).

4.4.1. Effect of flame locution. The effect of the flame location on the flame speed is shown in Fig. 14, where the difference in the flame locations results in an up to $20 \%$ difference in the flame speed. Figure 14 also shows that, within the pore, two flame positions are possible for the same flame speed. Due to the connectivity of the particles, a higher flame speed results and, for the same conductivity, an increase of $90 \%$ in the flame speed occurs when $c / d=0.1$ (compared to $c / d=0$ ). This is due to an increase in the preheat in the axial direction and an increase in conduction in the transverse direction. The increase in conduction in the transverse direction causes the temperature of the gas at $y / l=0.5$ to be very close to that of the solid, as shown in Fig. 13. The amount of preheat depends significantly on the position of the flame with respect to the transverse connections. When the flame is located downstream of the transverse connection (i.e. $x_{F} / l=0.5$ ), the preheating is enhanced significantly. This is shown in Fig. 13, where the temperatures at $y / l=0$ and 0.5 are nearly the same near the transverse connecting arm. IIowever, when the flame location is near the transverse connection (i.e. $x_{\mathrm{F}} / l \simeq 0$ ), the preheat is reduced since the upstream transverse connection is far from the reaction zone [i.e. $\left.\left(x-x_{\mathrm{F}}\right) / l=-1\right]$. The flame thickness and the excess temperature increase with increase in $x_{\mathrm{F}}$ $\left(\Delta T_{\max }^{*}=0.26\right.$ for $x_{\mathrm{F}} / l=0$ to 0.30 for $\left.x_{\mathrm{F}} / l=0.33\right)$.

The predictions of the volume-averaged models for the flame speed are also shown in Fig. 14. The twomedium treatment predicts a higher $u_{\mathrm{F}} / u_{\mathrm{F}_{\mathrm{o}}}$ compared to the two-dimensional solution (for all flame locations within the pore). This difference varies (with 
Table 5. Effect of the particle arrangement on the flame speed and the dimensionless excess temperature, $k_{\mathrm{s}} / k_{\mathrm{g}}=100$ and $\varepsilon=0.9$

\begin{tabular}{ccccc}
\hline & \multicolumn{4}{c}{$u_{\mathrm{F}} / u_{\mathrm{F}_{\mathrm{o}}}\left(\Delta T_{\max }^{*}\right)$} \\
\cline { 2 - 3 } Arrangement & \multicolumn{2}{c}{ Two-dimensional } & \\
\cline { 2 - 3 } & $x_{\mathrm{F}} / l=0$ & $x_{\mathrm{F}} / l=0.5$ & Two-medium & Single-medium \\
\hline \multirow{2}{*}{ Staggered } & 1.07 & 1.00 & 1.13 & 1.12 \\
& $(0.131)$ & $(0.005)$ & $(0.013)$ & 1.21 \\
In-line & 1.04 & 0.95 & 1.22 & \\
& $(0.125)$ & $(0.038)$ & $(0.021)$ & \\
\hline
\end{tabular}

Table 6. Effect of the equivalence ratio on the flame speed and the dimensionless excess temperature, in-line arrangement, $k_{\mathrm{s}} / k_{\mathrm{g}}=100$, and $\varepsilon=0.9$

\begin{tabular}{ccccc}
\hline & \multicolumn{4}{c}{$u_{\mathrm{F}} / u_{\mathrm{F}_{\mathrm{o}}}\left(\Delta T_{\mathrm{max}}^{*}\right)$} \\
\cline { 2 - 3 }$\Phi$ & \multicolumn{2}{c}{ Two-dimensional } & & \\
\cline { 2 - 3 } & $x_{\mathrm{F}} / l=0$ & $x_{\mathrm{F}} / l=0.5$ & Two-medium & Single-medium \\
\hline \multirow{2}{*}{0.7} & 1.20 & 0.99 & 1.21 & 1.12 \\
& $(0.051)$ & $(0.014)$ & $(0.027)$ & 1.21 \\
1 & 1.04 & 0.95 & 1.22 & \\
& $(0.125)$ & $(0.038)$ & $(0.021)$ & \\
\hline
\end{tabular}

the flame position) and ranges between 2 and $30 \%$. The single-medium model predicts a higher flame speed, compared to the two-medium model. In the single-medium model, since the effective conductivity and mass diffusivity of the gas are much larger than that for the two-medium treatment, this results in a larger flame speed predicted by the single-medium model.

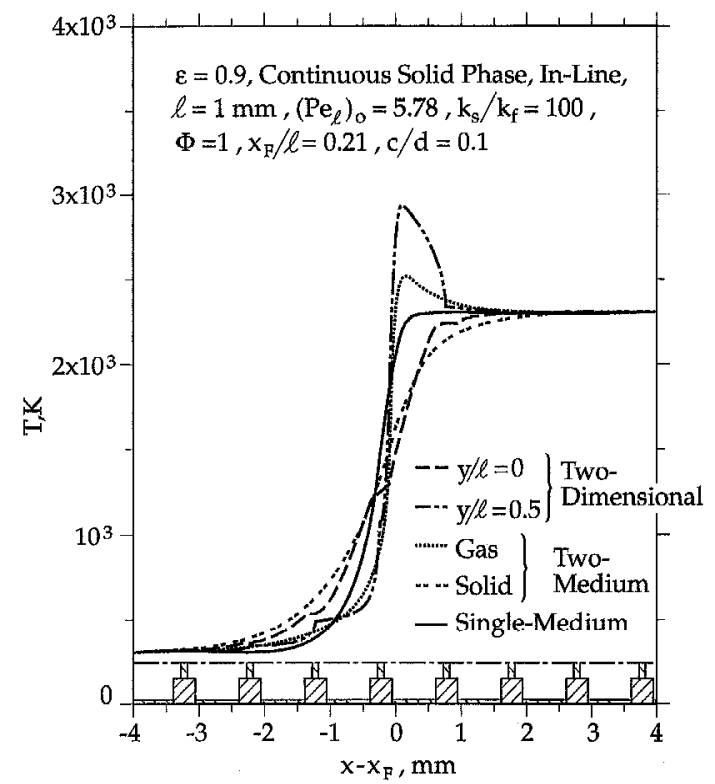

Fig. 13. Axial temperature distribution as predicted from the two-dimensional solutions and the single- and two-medium models, for connected square cylinders with the in-line arrangement.
4.4.2. Effect of $\mathrm{k}_{s} / k_{g}$. As the conductivity of the solid phase is increased, the conduction through the solid and the connecting arms increases, and, therefore, the flame speed also increases as shown in Fig. 15. For $k_{\mathrm{s}} / k_{\mathrm{g}}=1$, the flame speed is the same as that obtained from the discontinuous solid phase. The increase in

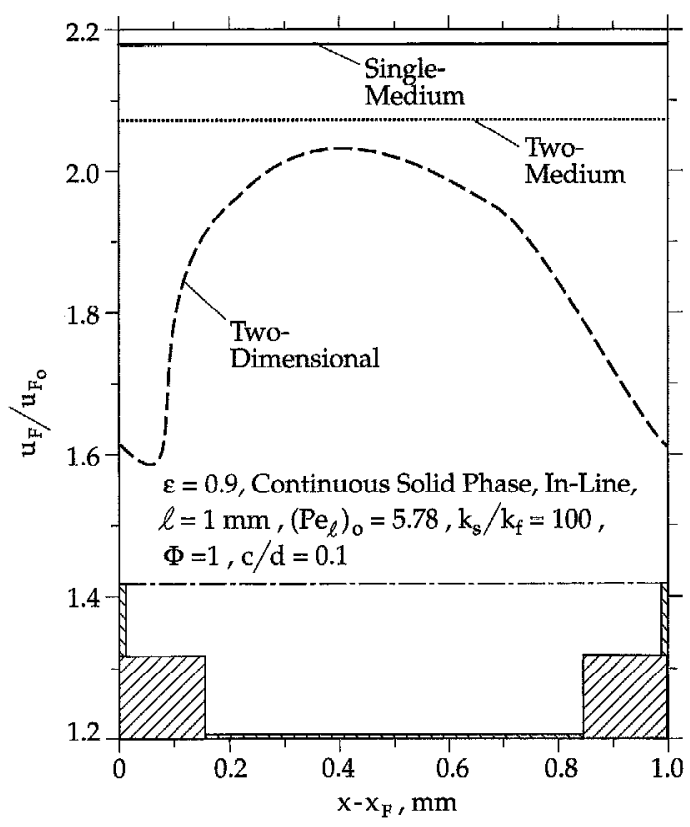

Fig. 14. Variation of the normalized flame speed with respect to the flame position for the continuous solid phase. The predictions of the single- and two-medium models are also shown. 


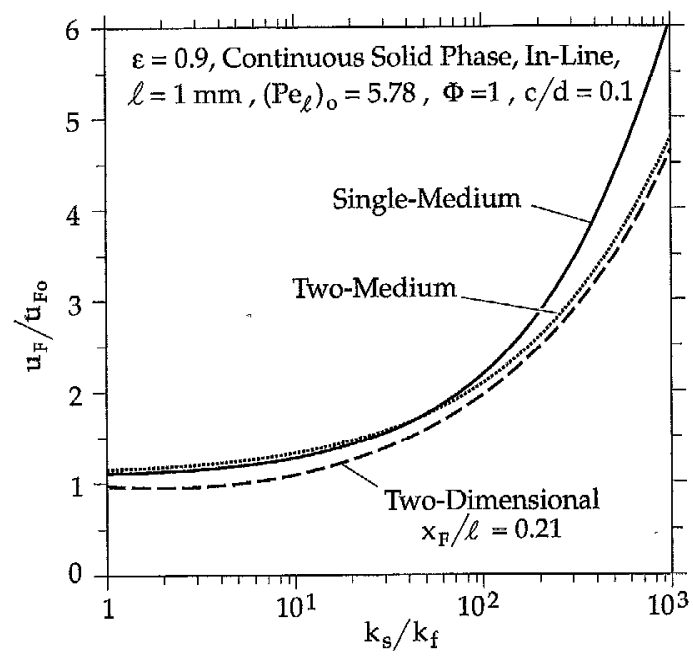

Fig. 15. Effect of the solid-phase conductivity on the normalized flame speed for the continuous solid phase. The predictions of the two-dimensional, single-medium and twomedium models are shown.

the flame speed due to the increase in the solid conductivity becomes about $350 \%$ when $k_{\mathrm{s}} / k_{\mathrm{g}}=1000$. Note that the solid phase effective conductivity for $k_{\mathrm{s}} / k_{\mathrm{g}}=1000$ is about 100 times larger than that for $k_{\mathrm{s}} / k_{\mathrm{g}}=1$. Also shown in Fig. 15 are the predictions from the volume-averaged models. Flame speed predictions of the two-medium treatment are in a closer agreement with the two-dimensional results. The single-medium treatment predicts a larger flame speed. The excess temperature also increases with the increase in the solid-phase conductivity. For example, for $k_{3} / k_{3}=1$ the two-dimensional solutions and the two-medium model give $\Delta T_{\max }^{*}=0.065$ and 0.013 , and for $k_{\mathrm{s}} / k_{\mathrm{g}}=100$ the results are 0.320 and 0.106 .

\section{CONCLUSIONS}

A two-dimensional, direct numerical simulation of adiabatic, premixed gaseous (methane-air) combustion in a porous medium made of in-line or staggered arrangements of discrete or connected square cylinders was made. The results for the flame speed and the temperature distribution are compared with those obtained from the volume-averaged models (i.e. single- and two-medium treatments). The two-dimensional results, which are for a flame thickness nearly the same as the pore size, show that within a pore multiple flame speeds exist. The two-dimensional results for the discrete solid phase show that:

(a) the flame speed is affected by the local hydrodynamics for $k_{\mathrm{s}} / k_{\mathrm{g}}=1$ and, as $k_{\mathrm{s}} / k_{\mathrm{g}}$ increases and when the particle is within the reaction zone, the preheat becomes more significant and the flame speed increases;

(b) as the porosity decreases the flame speed decreases for $k_{\mathrm{s}} / k_{\mathrm{g}}=1$ due to the velocity gradient within the pore and as $k_{\mathrm{s}} / k_{\mathrm{g}}$ increases the flame speed increases with a decrease in porosity;

(c) the flame speed is affected by the pore size and, as the pore size decreases, the flame encloses more solid and a higher flame speed results for $k_{\mathrm{s}} / k_{\mathrm{g}}>1$, and the effect of equivalence ratio $\Phi$ is similar since decreasing $\Phi$ increases the flame thickness and also the excess temperature decreases;

(d) the flame speed is affected by the particle arrangement since the local hydrodynamics is altered, and, for the same porosity and solid phase conductivity, the flame speed is larger for the staggered arrangement; and

(e) the normalized flame speed obtained from the two-dimensional simulations ranges between 0.75 and 1.1 and the maximum dimensionless excess temperature found is about 0.2 , while the two-medium treatment predicts a higher flame speed and a lower excess tempcraturc.

The results for the continuous solid phase show that:

(a) the flame speed becomes much higher once the particles are connected, and, for the two-dimensional model porous medium used, the normalized flame speed approaches a magnitude of about 5 and the dimensionless excess temperature reaches 0.55 , for $k_{\mathrm{s}} / k_{\mathrm{f}}=1000$;

(b) the variation of the flame speed within the pore becomes more pronounced; and

(c) the two-medium model predicts flame speeds that are higher by as much as $20 \%$ and the predicted excess temperature is lower.

The radiative heat transfer can significantly affect the flame speed and the extent of the local thermal nonequilibrium. This and more realistic descriptions of the solid-phase distribution are needed in order to make the results directly usable for practical analysis and design.

Acknowledgement-The authors would like to thank Professor K. Hanamura for his valuable suggestions.

\section{REFERENCES}

1. R. Echigo, Radiation enhanced/controlled phenomena of heat and mass transfer in porous media, ASME/JSME Thermal Engineering Joint Conference, Vol. 4, pp. xxi-xxxii (1991).

2. K. Hanamura, R. Echigo and S. A. Zhdanok, Superadiabatic combustion in porous media, Int.J. Heat Mass Transfer 36, 3201-3209 (1993).

3. T. Takeno and K. Sato, An excess enthalpy flame theory, Combust. Sci. Technol. 20, 73-84 (1979).

4. T. Takeno, K. Sato and K. Hase, A theoretical study on an excess enthalpy flame, Eighteenth Symposium (International) on Combustion, pp. 465-472. Combustion Institute (1981).

5. T. Takeno and K. Hase, Effects of solid length and heat loss on an excess enthalpy flame, Combust. Sci. Technol. 31, 207-215 (1983).

6. Y. Yoshizawa, K. Sasaki and R. Echigo, Analytical 
study of the structure of radiation controlled flame, Int J. Heat Mass Transfer 31, 311-319 (1988).

7. S. B. Sathe, R. E. Peck and T. W. Tong, A numerical analysis of heat transfer and combustion in porous radiant burners, Int. J. Heat Mass Transfer 33, 1331-1338 (1990).

8. S. B. Sathe, R. E. Peck and T. W. Tong, Flame stabilization and multimode heat transfer in inert porous media : a numerical study, Combust. Sci. Technol. 70, 93-109 (1990).

9. M. Kaviany, Principles of Convective Heat Transfer. Springer-Verlag, New York (1994).

10. M. Kaviany, Principles of Heat Transfer in Porous Media. Springer-Verlag, New York (1991).

11. M. Sahraoui and M. Kaviany, Slip and no-slip temperature boundary conditions at interface of porous, plain media : conduction, Int. J. Heat Mass Transfer 36, 1019-1033 (1993).
12. M. Sahraoui and M. Kaviany, Slip and no-slip temperature boundary conditions at interface of porous, plain media : convection, Int. J. Heat Mass Transfer 37, 1029-1044 (1994)

13. M. Kaviany and B. P. Singh, Radiative heat transfer in porous media, Adv. Heat Transfer 23, 133-186 (1993).

14. I. Nozad, R. G. Carbonell and S. Whitaker, Heat conduction in multi-phase systems $I$ : theory and experiments for two-phase systems, Chem. Engng Sci. 40, 843855 (1985).

15. S. V. Patankar, Numerical Heat Transfer and Fluid Flow. Hemisphere, Washington, DC (1980).

16. F. Mauss and N. Peters, Reduced kinetic mechanisms for premixed methane-air flames. In Reduced Kinetic Mechanisms for Application in Combustion Systems (Edited by N. Peters and B. Rogg), pp. 58-75. SpringerVcrlag, Berlin (1993). 